\title{
Nutzen Auszubildende bei der Bearbeitung berufsfeldbezogener Mathematikaufgaben ihr Wissen aus der Schule? Eine qualitative Untersuchung mit angehenden Industriekaufleuten
}

\author{
Robert von Hering (D) A Anja Rietenberg • Aiso Heinze $\mathbb{D}$ • \\ Anke Lindmeier iD
}

Eingegangen: 10. Dezember 2019 / Angenommen: 6. Januar 2021 / Online publiziert: 25. Januar 2021 (C) Der/die Autor(en) 2021

Zusammenfassung Mathematischen Kompetenzen wird eine wichtige Rolle für den erfolgreichen Übergang von der allgemeinbildenden Schule in die berufliche Erstausbildung zugesprochen. Wie in der Schule erworbene mathematische Kompetenzen beim Übergang in der beruflichen Ausbildung Wirkung zeigen, ist bis auf statistische Zusammenhänge allerdings wenig untersucht. Erschwert wird die Forschung u. a. dadurch, dass auf beiden Seiten grundsätzlich verschiedene und nicht unmittelbar anschlussfähige Kompetenzbegriffe genutzt werden. Während Anforderungen im Mathematikunterricht der allgemeinbildenden Schule allgemeinbildende mathematische Kompetenzen adressieren und diese eher an einer Wissensbildung orientiert sind, werden berufliche Kompetenzen zur Bewältigung realer beruflicher Anforderungen benötigt und sind somit eher handlungsorientiert zu verstehen. In der Forschung wurde das Konstrukt berufsfeldbezogene mathematische Kompetenzen als eine vermittelnde Größe zwischen den verschiedenen Kompetenzkonstrukten vorgeschlagen, das als Ansatzpunkt für wissenschaftliche Studien zum Übergang Schule - Beruf genutzt werden kann. Darauf aufbauend untersuchen wir in der vorliegenden empirischen Studie, inwiefern sich solche berufsfeldbezogenen mathematischen Kompetenzen von allgemeinbildenden mathematischen Kompetenzen abgrenzen lassen. Aufgrund der inhaltlichen Nähe zum Unterrichtsfach Mathematik haben wir exemplarisch das Berufsfeld der Industriekaufleute gewählt. Für eine Stimulated-Recall-Interviewstudie wurden Aufgabenpaare entwickelt, die bei ma-

Zusatzmaterial online Zusätzliche Informationen sind in der Online-Version dieses Artikels (https:// doi.org/10.1007/s13138-021-00181-8) enthalten.

R. von Hering $(\bowtie) \cdot$ A. Heinze $\cdot$ A. Lindmeier

IPN - Leibniz-Institut für die Pädagogik der Naturwissenschaften und Mathematik, Olshausenstraße 62, 24118 Kiel, Deutschland

E-Mail: hering@leibniz-ipn.de

A. Lindmeier

Friedrich-Schiller-Universität Jena, Ernst-Abbe-Platz 2, 07743 Jena, Deutschland 
thematisch-inhaltlicher Parallelität die unterschiedlichen Grade an beruflicher Anforderung widerspiegeln. Die Auswertung von Interviews mit 18 Auszubildenden zeigt, dass die Mathematikaufgaben mit berufsbezogenem bzw. allgemeinbildendem Kontext tatsächlich unterschiedliche kognitive Bearbeitungsprozesse hervorrufen. Die Ergebnisse lassen sich als qualitative Unterschiede zwischen allgemeinbildenden mathematischen und berufsfeldbezogenen mathematischen Kompetenzen interpretieren. Mögliche Implikationen für die Forschung und die Schulpraxis werden diskutiert.

Schlüsselwörter Berufsvorbereitung · Übergang Schule - Beruf · Berufliche Kompetenzen · Mathematische Kompetenzen · Industriekaufleute in der Ausbildung

\title{
Do Trainees use Their Knowledge from School when Dealing with Vocation-related Mathematical Problems? A Qualitative Study with Trainees as Industrial Clerks
}

\begin{abstract}
Mathematical competencies play a crucial role in the successful transition from general to vocational education. Little research has been done on the statistical connections, of how mathematical competencies acquired at school have an impact on the transition to vocational training. Research is made more difficult by the fact that fundamentally different and not directly connectable concepts of competence are used on both sides. While requirements in mathematics in general education school address general mathematical competencies and these are more oriented towards knowledge development, vocational competencies are required to cope with real vocational requirements and are therefore to be understood as more action-oriented. In research, the construct of vocation-related mathematical competencies has been proposed as a mediating factor between the different competence constructs in school and at work, which can be used as a starting point for scientific studies in the transition phase. Based on this, we investigate in the present empirical study to what extent such vocation-related mathematical competencies can be distinguished from general mathematical competencies. Due to the content-related proximity to the subject mathematics, we have chosen the vocational field of industrial clerks as an example. For a stimulated recall interview study, pairs of problems were developed that reflect the different degrees of vocational requirements in the case of parallel mathematical content. The evaluation of interviews with 18 trainees shows that the mathematical problems with a vocation-related or a general education context actually cause different cognitive processes. The results can be interpreted as qualitative differences between general mathematical and vocation-related mathematical competencies. Possible implications for research and school are discussed.
\end{abstract}

Keywords Vocational preparation - Transition from school to work - Vocational competencies · Mathematical competencies · Industrial clerk trainees 


\section{Einleitung \& Motivation}

Ein Ziel des allgemeinbildenden Schulunterrichts besteht darin, die Schülerinnen und Schüler auf ihr Berufsleben vorzubereiten (KMK 2017a). Auch in den Bildungsstandards Mathematik für den Mittleren Schulabschluss sowie für die Allgemeine Hochschulreife bilden sich als Teil der Mathematical Literacy (Neubrand 2003) Ziele der fachlichen Berufsvorbereitung sowie der Berufsorientierung ab (KMK 2003, 2012). Trotz dieser Rahmenbedingungen stoßen Ansätze zur Untersuchung von mathematischen Kompetenzen im Übergang von der allgemeinbildenden Schule in die berufliche Ausbildung und auch zum weiteren Ausbildungsverlauf auf konzeptionelle Schwierigkeiten (vgl. OECD 2000), die wesentlich mit der Konzeptualisierung des Begriffs Kompetenz zu tun haben. Eine besondere Herausforderung besteht darin, mathematische Kompetenz so zu beschreiben, dass sie einerseits als valide hinsichtlich der Vergleichbarkeit der Phasen vor, während und nach dem Übergang angesehen werden kann und andererseits als valide für jede Phase an sich (bzw. zumindest für die Phase vor und nach dem Übergang). Jedoch folgen die Zielvorgaben für die schulische (Bildungsstandards und Lehrpläne) und berufliche Bildung (Rahmenlehrpläne der Ausbildungsberufe) unterschiedlichen Logiken. Insbesondere unterscheiden sich die Kompetenzbegriffe, die in der allgemeinbildenden Schule und in der beruflichen Ausbildung zugrunde gelegt werden gemäß den sehr unterschiedlichen Zielsetzungen der Bildungsphasen (Erpenbeck 2009). Um dieser Herausforderung zu begegnen, wurden für den Bereich der Mathematik theoretische Modelle entwickelt, um die spezifischen mathematischen Anforderungen aus Schule und Beruf aufeinander zu beziehen (Winther et al. 2013; Neumann et al. 2013). Ziel dieser Ansätze ist es, den Übergang Schule - Beruf auch mit Blick auf eine kumulative Kompetenzentwicklung besser beschreiben zu können. Gleichzeitig sollen die Modelle es dadurch vereinfachen, Leistungen aus der allgemeinbildenden Schule, der beruflichen Ausbildung und dem Beruf hinsichtlich ihrer Einfluss- und Erfolgsfaktoren zu untersuchen und die Befunde zu interpretieren.

So wurden schulische mathematische Kompetenzen in größeren empirischen Studien wiederholt als zentrale Größe für einen erfolgreichen Übergang in die Berufsausbildung identifiziert (z. B. Lehmann und Seeber 2007). Auch wenn diese statistischen Zusammenhänge eine hohe inhaltliche Plausibilität haben, so sind vertiefte Analysen zur weiteren Aufklärung erstrebenswert. Insbesondere gilt es aus einer fachspezifischen Perspektive heraus, die konkreten Herausforderungen zu verstehen, die Auszubildende beim Nutzen ihrer schulisch erworbenen mathematischen Kompetenz in beruflichen Anwendungskontexten erfahren. Das von Neumann et al. (2013) vorgeschlagene Kompetenzmodell kann dazu geeignet sein. Anknüpfend an die unterschiedlichen mathematischen Anforderungen und die differierenden Kompetenzbegriffe in der allgemeinbildenden Schule und in der (kaufmännischen) Berufsausbildung wird hier das Konstrukt der berufsfeldbezogenen mathematischen Kompetenzen als potenziell vermittelnder Kompetenzbereich am Übergang in die Berufsausbildung beschrieben. Dabei handelt es sich um mathematische Kompetenzen, die einerseits spezifisch für die Bewältigung beruflicher oder berufsnaher Anforderungen benötigt werden und die andererseits als Teil der schulisch erworbenen mathematischen Kompetenzen angesehen werden können. Bisher steht eine 
vertiefte Prüfung aus, inwiefern sich diese theoretischen Annahmen auch empirisch abbilden lassen.

Für derartige Studien ist es zweckmäßig, sich zunächst auf Ausbildungsberufe zu fokussieren, in denen mathematische Kompetenzen von zentraler Bedeutung sind. Die Rahmenlehrpläne der Ausbildungsberufe zeigen, dass insbesondere Kaufleute und speziell Industriekaufleute in der Berufsausbildung mit vergleichsweise hohen mathematischen Anforderungen konfrontiert sind (z. B. KMK 2002). Diese Berufe stehen daher - und wegen ihrer hohen Anzahl an Auszubildenden - im Mittelpunkt unserer Aufmerksamkeit. In der im Folgenden berichteten Interviewstudie untersuchen wir konkret, ob Auszubildende zu Industriekaufleuten, die gerade den Übergang Schule - Beruf erleben, unterschiedliche Kompetenzen nutzen, wenn sie parallele mathematische Probleme in einem beruflichen und einem nichtberuflichen Kontext bearbeiten. Auf diese Weise wollen wir insbesondere ergründen, ob allgemeinbildende mathematische Kompetenzen und berufsfeldbezogene mathematische Kompetenzen empirisch voneinander abgrenzbar sind oder nicht und inwieweit sich die von Neumann et al. (2013) vorgeschlagene theoretische Modellierung zur differenzierteren Beschreibung von mathematischen Kompetenzen in der Übergangsphase Schule - Beruf als tragfähig herausstellt.

\section{Theoretischer Hintergrund}

\subsection{Die berufliche Ausbildung (der Industriekaufleute) in Deutschland}

Die berufliche Ausbildung in Deutschland ist durch das Berufsbildungsgesetz (BBiG 2005) geregelt. Es sieht mit der betrieblichen Berufsbildung, den beruflichen Schulen sowie den außerbetrieblichen Ausbildungseinrichtungen drei unterschiedliche Lernorte vor (BBiG 2005, § 2), wobei die letzten beiden zusammengefasst werden, wenn der Begriff des Dualen Systems verwendet wird. Diese Ausbildungsstruktur zeichnet sich durch die Verzahnung von Theorie (berufliche Schule) und Praxis (Betrieb) aus und hat weltweit ein hohes Ansehen (Jongebloed 2011). Die Grundlage bilden für jeden Ausbildungsberuf zum einen die jeweilige Ausbildungsordnung für die betriebliche Ausbildung und zum anderen der jeweilige Rahmenlehrplan für die (berufs-)schulische Ausbildung (BBiG 2005). In der berufsschulischen Ausbildung organisiert sich der Unterricht - abhängig vom jeweiligen Ausbildungsberuf in klassischen allgemeinbildenden Unterrichtsfächern und daneben in spezifischeren Unterrichtsfächern mit konkretem Bezug zum Ausbildungsberuf, beispielsweise Rechnungswesen im Rahmen der Ausbildung für Industriekaufleute (KMK 2002).

Am Übergang von der allgemeinbildenden Schule in die berufliche Ausbildung treten strukturelle Probleme auf, die erst teilweise verstanden werden. Es wird beispielsweise eine steigende Anzahl unbesetzter Lehrstellen sowie eine recht konstante Anzahl an Jugendlichen, die erfolglos einen Ausbildungsplatz suchen, beobachtet, was ein Teil des sogenannten Passungsproblems ist (BIBB 2019). Gleichzeitig konnte herausgearbeitet werden, dass die von den Betrieben angegebenen notwendigen Qualifikationen für eine Ausbildung geringer sind als die tatsächlichen Anforderungen, die an die Auszubildenden gestellt werden (Münk 2013). Außerdem werden 
etwa ein Viertel der Ausbildungsverträge im Laufe der Ausbildung aufgelöst, wobei die Jugendlichen vermehrt auch Faktoren wie Uninformiertheit über Inhalt und Aufbau der Ausbildung und fehlerhafte Berufsbilder und -vorstellungen als Ursache angeben (BIBB 2019). Das bundesweite Berufsorientierungsprogramm BOP versucht daher auf der Basis des Modells zur Berufswahlkompetenz von Ratschinski et al. (2018) Berufsbilder und -vorstellungen transparent zu machen und diese den Jugendlichen zu vermitteln, sodass Schülerinnen und Schüler u. a. zur erfolgreichen und eigenverantwortlichen Gestaltung des Übergangsprozesses in eine Ausbildung befähigt werden (KMK 2017a). Die allgemeine berufliche Orientierung wird dabei als fächerübergreifender Auftrag für allgemeinbildende Schulen verstanden (KMK 2017b).

Mit Blick auf den Beitrag des allgemeinbildenden Mathematikunterrichts zur Berufsvorbereitung kann der kaufmännische Ausbildungsbereich im Allgemeinen und der Beruf Industriekaufmann/-frau im Speziellen als repräsentative Berufsgruppe gesehen werden, da er ein besonders relevanter Ausbildungsbereich ist und zudem vergleichsweise hohe mathematische Anforderungen an die Auszubildenden stellt. Da dieser Ausbildungsberuf auch für unsere Studie herangezogen wurde, soll er im Folgenden detaillierter vorgestellt werden.

Die meisten Ausbildungsplätze fallen in Deutschland in den Wirtschaftssektor Industrie \& Handel. Im Jahr 2018 kamen 58\% der neu abgeschlossenen Ausbildungsverträge aus diesem Sektor, gefolgt vom Sektor Handwerk mit $27 \%$ aller Ausbildungsverträge (BIBB 2019). Seit Jahren befindet sich der Ausbildungsberuf Industriekaufmann/-frau neben drei weiteren kaufmännischen Berufen unter den fünf Berufen mit den meisten neu abgeschlossenen Ausbildungsverträgen (BIBB 2019). Die dreijährige Ausbildung findet dual und gleichberechtigt in Betrieb und beruflicher Schule statt und ist durch den Rahmenlehrplan der KMK und die Ausbildungsordnungen des Bundes für die betriebliche Ausbildung geregelt (KMK 2002; BMBF 2002). Über alle Ausbildungsberufe hinweg ist dieser Ausbildungsberuf der Beruf mit der höchsten Anzahl und einem der höchsten Anteile (71\% in 2017) an Auszubildenden mit Hochschulzugangsberechtigung (BIBB 2019).

Aus mathematikdidaktischer Sicht ist die Ausbildung der Industriekaufleute von Interesse, da sie einen hohen Anteil an Lerninhalten mit mathematischem Gehalt aufweist. Im Rahmenlehrplan für die berufsschulische Ausbildung der Industriekaufleute (KMK 2002) weisen fünf der zwölf Lernfelder einen starken mathematischen Bezug auf (von Hering et al. 2020). Die mathematisch relevanten Inhalte dieser fünf Lernfelder lassen sich aus inhaltlicher Sicht in die vier Kategorien Kostenrechnung, Leistungsrechnung, Abschreibung sowie Finanzierung und Investition bündeln. Aus Perspektive der Mathematik handelt es sich bei den in den Lernfeldern verankerten Inhalten schwerpunktmäßig um den Umgang mit linearen Zusammenhängen, speziell Anwendungen von Dreisatz und Prozentrechnung. Diese Inhalte werden in der allgemeinbildenden Schule in der Sekundarstufe I unterrichtet. Der Unterschied zwischen der Anwendung dieser Inhalte in der Sekundarstufe I und in der Ausbildung der Industriekaufleute findet sich in der jeweiligen Zielsetzung: In der allgemeinbildenden Schule liegt der Fokus im Erwerb einer auch disziplinär verorteten mathematischen Grundbildung, die unter anderem zur Umwelterschließung und für das Problemlösen (durchaus in verschiedenen Kontexten) befähigen soll 
(Winter 1995). In der Ausbildung hingegen liegt der Fokus auf der Bewältigung von beruflichen Anforderungen unter (häufig impliziter) Nutzung mathematischer Mittel. Das heißt, Mathematik wird in der Ausbildung immer innerhalb von beruflichen Kontexten angewendet und anders als in der Schule sind die Strukturierungen der Lerninhalte nicht fachsystematisch geprägt (z. B. linearer Zusammenhang), sondern vom beruflich Kontext (z. B. Abschreibung, Stückkosten). Entsprechend kommt der kaufmännischen Fachsprache (z.B. Break-Even-Point) im Vergleich zur Schulmathematik eine hohe Bedeutung zu (vgl. von Hering et al. 2020).

Zusammengefasst ist Industriekaufmann/-frau ein Beruf, für den häufig ausgebildet wird und der zudem eine besondere Nähe zum Schulfach Mathematik aufweist. Viele Anforderungen im Ausbildungsverlauf bauen inhaltlich auf den allgemeinbildenden mathematischen Kompetenzen auf, die während der Schulzeit erworben wurden. Dieser Beruf bietet sich also an, um die Unterschiede beider Systeme (allgemeinbildende Schule vs. berufliche Ausbildung) in Bezug auf die vorkommenden mathematischen Anforderungen und zugehörigen Kompetenzen exemplarisch $\mathrm{zu}$ analysieren. Basis dafür ist das im folgenden Abschnitt erläuterte theoretische Kompetenzmodell von Neumann et al. (2013) zum Übergang von der allgemeinbildenden in die berufliche Schule aus Mathematik-Sicht.

\subsection{Berufsfeldbezogene mathematische Kompetenzen}

Beim Eintritt in die berufliche Erstausbildung ändern sich die Anforderungen an die Lernenden im Vergleich zur allgemeinbildenden Schule in vielerlei Hinsicht. Neben der neuen Lernsituation im Dualen System ist insbesondere auch die Art der zu erwerbenden Kompetenzen unterschiedlich. Dies hat Auswirkungen auf den Verlauf des individuellen Kompetenzerwerbs, sodass nicht zwangsläufig von einem kontinuierlichen, kumulativen Kompetenzaufbau am Übergang von der allgemeinbildenden Schule in die berufliche Erstausbildung ausgegangen werden kann (Heinze und Grüßing 2009; Neumann et al. 2013). Im Folgenden soll daher mit Hilfe der unterschiedlichen Kompetenzbegriffe der allgemeinbildenden Schule bzw. der beruflichen Bildung herausgearbeitet werden, auf welche Weise sich deren nicht unmittelbare Anschlussfähigkeit in der Übergangsphase äußert.

Für den beruflichen Kontext beschreibt Nickolaus (2011) auf Basis des allgemeinbildenden Kompetenzbegriffs nach Weinert (2001) den Begriff der Handlungskompetenz als orientierend, wobei sich das Handeln auf komplexe berufliche Anforderungssituationen bezieht. Ziel der Berufsausbildung ist u.a. der Erwerb einer beruflichen Handlungskompetenz und damit die Entwicklung einer beruflichen Mündigkeit (Nickolaus und Seeber 2013). Auch die KMK (2011) verwendet für die Zielsetzung der berufsschulischen Ausbildung das Konstrukt der beruflichen Handlungskompetenz als ein berufsspezifisch ausgestaltetes Konzept, wobei stets kontextualisierte und situationsgebundene Anforderungen vorkommen. Erpenbeck (2009, S. 18) bezeichnet den dargestellten Kompetenzbegriff der beruflichen Bildung als „Performanzrichtung“ des Kompetenzbegriffs und grenzt sie von der „Kognitionsrichtung“ des Kompetenzbegriffs der allgemeinen Bildung ab. Letztere bezeichnet die Nutzung von Kompetenzen, die auf die Bewältigung domänenspezifischer 
Anforderungen ${ }^{1}$ abzielt und eher der Wissensbildung dient (Erpenbeck 2009). Dabei werden Kompetenzen als „,kontextspezifische kognitive Leistungsdispositionen, die sich funktional auf Situationen und Anforderungen in bestimmten Domänen beziehen“ (Klieme und Leutner 2006, S. 879), aufgefasst. Sie beziehen sich auf den Erwerb fachlich strukturierter kognitiver Grundlagen im Sinne des allgemeinbildenden Auftrags von Schule und tragen damit zur Bildung im Sinne einer Grundbildung bei. Zwar wird die breite Anwendbarkeit der erworbenen Kompetenzen angepeilt, gleichzeitig aber der Erwerb der zugrundeliegenden Wissensstrukturen betont, was sich beispielsweise in den Bildungsstandards Mathematik (KMK 2003, 2012) und dem PISA zugrunde liegenden Konzept der Mathematical Literacy (Neubrand 2003) abbildet.

In ihrem integrierten Kompetenzmodell für die kaufmännische Domäne liefern Winther et al. (2013) einen Ansatz aus berufsspezifischer Perspektive, wie die beiden Bereiche der schulischen und der beruflichen Kompetenzentwicklung aufeinander bezogen werden können. Hier wird aus theoretischer und normativer Sicht davon ausgegangen, dass allgemeine Kompetenzen die Grundlage der beruflichen Kompetenzen bilden und sich im Verlauf der beruflichen Ausbildung berufsspezifisch weiterentwickeln (Winther 2010). Berufliche Kompetenzen wiederum gliedern sich in eine domänenverbundene und eine domänenspezifische Komponente ${ }^{2}$. Die domänenverbundene Kompetenz beschreibt Aspekte einer berufsbezogenen kaufmännischen Grundbildung im Sinne eines kaufmännischen bzw. ökonomischen Vorwissens (Nickolaus und Seeber 2013). Zur Bewältigung domänenverbundener Anforderungen müssen schulisch erworbene Kenntnisse und Kompetenzen in kaufmännischen Anforderungssituationen reorganisiert werden (Winther et al. 2013), etwa, wenn mathematische Kompetenzen aus der Schule angewendet werden müssen, um einen ökonomischen Zusammenhang zu modellieren. Die domänenspezifische Kompetenz beschreibt die eigentliche berufsspezifische Fachkompetenz, welche sich auf die Bewältigung von typischen Anforderungen in der Domäne bezieht und - in Abgrenzung zur domänenverbundenen Komponente - domänenspezifisches Vorwissen erfordert (Winther und Achtenhagen 2008). Dieses berufsspezifische Modell nimmt also die realen beruflichen Anforderungen als Ausgangspunkt, um die Anforderungen am Übergang Schule - Beruf zu fassen. Das Modell umfasst daher in der Schule genutzte Kompetenzmodelle der Kognitionsrichtung nicht.

Hier setzen Neumann et al. (2013) an und modellieren die mathematischen Kompetenzen am Übergang in die berufliche Ausbildung theoretisch aus Sicht der schulischen (= allgemeinbildenden) Seite (vgl. Abb. 1). Sie grenzen darin im Anschluss an Erpenbeck (2009) ebenso allgemeinbildende mathematische Kompetenzen im Sinne der Kognitionsrichtung von den eben skizzierten beruflichen Kompetenzen im Sinne der Performanzrichtung ab. Dabei bezeichnen allgemeinbildende mathematische Kompetenzen die Kompetenzen, die in der allgemeinbildenden Schule erworben werden sollen und beispielsweise in den Bildungsstandards Mathematik (KMK 2003) konkretisiert sind. Im Kompetenzraster der Bildungsstandards werden die allgemeinbildenden mathematischen Kompetenzen über die drei Dimensionen

\footnotetext{
1 In diesem Kontext bezieht sich der Begriff „Domäne“ auf schulfachliche Strukturierungen.

2 In diesem Kontext bezieht sich der Begriff „Domäne“ auf berufliche Strukturierungen.
} 
Abb. 1 Zusammenhang zwischen den verschiedenen für die Kompetenzentwicklung in der beruflichen Ausbildung relevanten Kompetenzbereiche. (Nach Neumann et al. 2013)

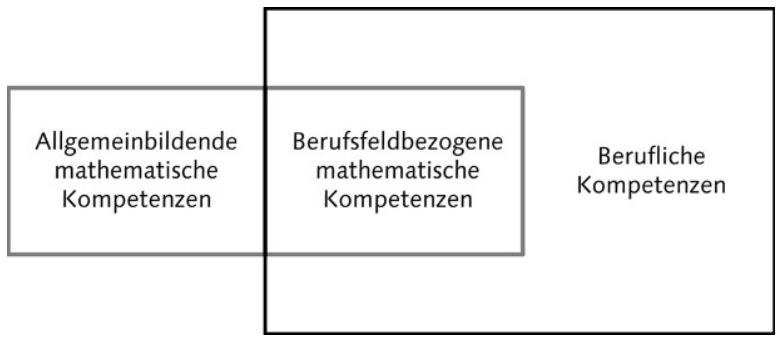

allgemeine mathematische Kompetenzen (Mathematisch argumentieren; Probleme mathematisch lösen; Mathematisch modellieren; Mathematische Darstellungen verwenden; Mit symbolischen, formalen und technischen Elementen der Mathematik umgehen; Mathematisch kommunizieren), mathematische Leitideen (Zahl; Messen; Raum und Form; Funktionaler Zusammenhang; Daten und Zufall) und Anforderungsniveau (Reproduzieren; Zusammenhänge herstellen; Verallgemeinern und Reflektieren) charakterisiert.

Als Kompetenzbereich, der sowohl den beruflichen als auch den allgemeinbildenden mathematischen Kompetenzen zuzuordnen ist, erscheint bei Neumann et al. (2013) der Bereich der berufsfeldbezogenen mathematischen Kompetenzen. Diese beziehen sich auf außerschulische, berufsnahe Anforderungssituationen, für deren Bewältigung jedoch vorwiegend allgemeinbildende mathematische Kompetenzen notwendig sind. Sie beschreiben damit solche Kompetenzen, die Auszubildende bis zum Beginn der Ausbildung durch den Mathematikunterricht an der Schule erworben haben sollten und die auch nach einer beruflich geprägten Kompetenzauffassung relevant sind. Die theoretische Klassifikation berufsfeldbezogener mathematischer Kompetenzen als Teil der allgemeinbildenden mathematischen Kompetenzen (und auch der beruflichen Kompetenzen) ist dabei entscheidend: Es handelt sich also nicht um einen von den allgemeinbildenden mathematischen Kompetenzen disjunkt abgrenzbaren Bereich. Die schematische Darstellung in Abb. 1 verdeutlicht dies in dem Sinne, dass berufsfeldbezogene mathematische Kompetenzen im Modell die Schnittmenge der allgemeinbildenden mathematischen Kompetenzen und der beruflichen Kompetenzen bilden.

Theoretisch können solche berufsfeldbezogenen mathematischen Kompetenzen, so sie in der Schule erworben wurden, am Übergang in die berufliche Ausbildung eine vermittelnde Rolle spielen. Neumann et al. (2013) argumentieren daher, dass mit Hilfe dieses Kompetenzbereichs die nicht unmittelbare Anschlussfähigkeit von fachlichen Bildungsprozessen im Übergang Schule - Beruf untersucht werden kann. Der forschungspraktische Nutzen dieses Modells ist jedoch noch nicht geklärt. Auch ist anzumerken, dass das Modell theoretisch erst einmal alle Domänen und Ausbildungsberufe als Berufsfeld zulässt, die für einzelne Studien spezifiziert werden müssen. Dies geschieht in unserer Untersuchung durch die Wahl des Ausbildungssektors Industrie \& Handel und des konkreten Ausbildungsberufs Industriekaufmann/-frau.

Für den gewerblich-technischen Ausbildungsbereich konnten Frank et al. (2017) auf der Grundlage des Modells von Neumann et al. (2013) bereits zeigen, dass allgemeinbildende physikalische Kompetenzen und berufsfeldbezogene physikalische 
Kompetenzen (beide analog $\mathrm{zu}$ den entsprechenden mathematischen Kompetenzen definiert) berufsspezifische Kompetenzen der jeweiligen Berufe vorhersagen. Im Rahmen einer Studie mit über 600 industriekaufmännischen Auszubildenden deuten erste statistische Analysen von Siebert und Heinze (2016) auf eine mögliche empirische Abgrenzbarkeit der allgemeinbildenden mathematischen und berufsfeldbezogenen mathematischen Kompetenzen hin. Damit würde in Frage gestellt, ob berufsfeldbezogene mathematische Kompetenzen in der allgemeinbildenden Schule erworben werden. Es fehlen bisher jedoch qualitative Analysen, die das Konstrukt der berufsfeldbezogenen mathematischen Kompetenzen in Abgrenzung zu allgemeinbildenden mathematischen Kompetenzen untersuchen. Die vorliegende Studie soll dies liefern und somit einen Beitrag zur Validierung des Konstrukts leisten.

\subsection{Die Rolle des Kontextes beim Erwerb mathematischer Kompetenzen}

Berufsfeldbezogene mathematische Kompetenzen werden u.a. als Teilbereich der allgemeinbildenden mathematischen Kompetenzen definiert. Die Unterscheidung wird entlang der beruflich relevanten vs. beruflich nicht relevanten Anforderungen getroffen. Dies wirft die Frage auf, ob es sich dabei, wie erste Ergebnisse (Siebert und Heinze 2016) andeuten, um voneinander empirisch abgrenzbare Kompetenzdimensionen handelt oder ob eine Unterscheidung entlang des Grades unterschiedlicher Berufsbezogenheit von Anforderungen praktisch irrelevant ist. Die beiden folgenden illustrativen Aufgabenbeispiele sollen als Ausgangspunkt für die Herangehensweise an diese Fragestellung dienen:

1. Die Arbeitslosenzahl in Neustadt betrug im vergangenen Jahr 2540 Personen. Nun ist sie um $3 \%$ gestiegen. Wie hoch ist die Arbeitslosenzahl in Neustadt jetzt?

2. Die Herstellungskosten für einen Computertisch betragen $220 €$. Ein zusätzlicher Verwaltungszuschlag beläuft sich auf $12 \%$. Was sind die realen Kosten eines solchen Computertisches?

Aus innermathematischer Sicht unterscheiden sich die beiden Aufgaben kaum voneinander. Zu einem bekannten Grundwert wird mit einem bekannten Prozentsatz der Prozentwert berechnet. Lediglich die Zahlenwerte unterscheiden sich, was den Situationen geschuldet ist. Geht man bei der Betrachtung über die mathematische Struktur hinaus, ergibt sich ein differenzierteres Bild. Für beide Aufgaben müssen beim Lösen aus den Texten Situationen entnommen werden. Während bei der ersten Aufgabe ein allgemeingesellschaftlich relevantes Phänomen (Arbeitslosenzahl) verstanden werden muss, ist in der zweiten Aufgabe eine kaufmännische Situation zu rekonstruieren. Der Text der zweiten Aufgabe verwendet kaufmännisch geprägte Begriffe (Kosten, Zuschlag), die zwar mit schulischem Wissen erschließbar sind, aber kaufmännische Fachsprache spiegeln (reale Kosten). Deswegen lässt sich die zweite Aufgabe im oben ausgeführten Sinne als berufsfeldbezogene mathematische Anforderung charakterisieren. Beide Aufgaben liegen aber trotz der Unterschiede klar im Anforderungsbereich der Bildungsstandards, sollten also mit allgemeinbildenden mathematischen Kompetenzen im Sinne der Bildungsstandards lösbar sein. Es stellt sich jetzt also die Frage, inwiefern Aufgaben, die sich auf so eine Weise unterscheiden, beim Lösen eventuell innerhalb der allgemeinbildenden mathemati- 
schen Kompetenzen gleiche (oder unterscheidbare) Kompetenzen erfordern, was in diesem Artikel als Frage nach der Abgrenzung berufsfeldbezogener mathematischer Kompetenzen von allgemeinbildenden mathematischen Kompetenzen gefasst ist.

Da sich die unterschiedlichen Anforderungen entlang der Kontexte manifestieren, hat die Frage einen Bezug zur kognitionspsychologischen Forschung und dort zum Transfer von Fähigkeiten. Die sogenannte Transferproblematik fasst die Beobachtung, dass die Übertragung von Fähigkeiten zwischen verschiedenen Kontexten schwierig ist (zsf. Perkins und Salomon 1992). Dies gilt vor allem dann, wenn sich die Kontexte wenig ähnlich sind (sog. ferner Transfer). In Zusammenhang damit konnten Cosmides und Tooby (1992) in Variationen eines psychologischen Experiments mit Hilfe der Wason selection task zeigen, dass Anforderungen besser bewältigt werden, wenn sie in bekannten (dort: sozialen) Kontexten auftreten. Dies weist darauf hin, dass für eine Person je nach Situation selbst strukturgleiche Anforderungen unterschiedlich erscheinen können. In diesem Verständnis ist also die Bewältigung einer Anforderung nicht losgelöst vom Kontext zu sehen, sondern die Situation ist integraler Bestandteil der jeweiligen Anforderung. Dies stützt bezogen auf die Fragestellung des Artikels die Differenzierung zwischen allgemeinbildenden mathematischen und berufsfeldbezogenen mathematischen Anforderungen.

Eine Erklärung für die beobachteten Transferproblematiken bietet u. a. die $\mathrm{Cog}$ nitive flexibility theory (Spiro et al. 1988) an, die erfolgreiches Problemlösen auf die Qualität der erworbenen Kompetenzen und damit auf Merkmale des Erwerbsprozesses zurückführt: Werden bereits während des Kompetenzerwerbs unterschiedliche Anwendungssituationen eingesetzt, so führt dies zu einer höheren kognitiven Flexibilität, sodass eine größere Bandbreite von Anforderungen bewältigt werden kann. Noch stärkere Annahmen in Bezug auf den Einfluss des Erwerbsprozesses trifft die Situated cognition theory (Brown et al. 1989): Hier wird von einer in den Erwerbskontexten verankerten Situiertheit der erworbenen Kompetenzen ausgegangen, sodass Kompetenzen und ihre Anwendung nicht von den Erwerbskontexten getrennt werden können.

Verglichen mit den kognitionspsychologischen Erkenntnissen erscheint der Diskurs zur Rolle von Kontexten im Zusammenhang mit den Zielsetzungen des Mathematikunterrichts und ihres Erreichens eher ambivalent: Einerseits fußt der in den Bildungsstandards zentrale allgemeinbildende mathematische Kompetenzbegriff auf dem Konzept der Mathematical Literacy. Für deren Erwerb wird die Anwendung von Mathematik in realistischen Kontexten als erforderlich gesehen (Neubrand 2003). Im Rahmen der PISA-Studien werden etwa konkret fünf relevante Kontextbereiche unterschieden und in den Tests gleichermaßen berücksichtigt (persönlich, bildungsbezogen, beruflich, öffentlich und wissenschaftlich, Deutsches PISA-Konsortium 2000). Andererseits wird jedoch die Bedeutung verschiedenartiger Kontexte und ihrer Rolle beim schulischen Kompetenzerwerb im Zusammenhang mit dem Konzept der Mathematical Literacy nicht weiter systematisch berücksichtigt. Beispielsweise werden selbst in den PISA-Studien keine differenzierten Analysen nach Kontextbereichen durchgeführt, um mögliche Effekte zu identifizieren.

Im engeren mathematikdidaktischen Diskurs werden Kontexte vor allem im Zusammenhang mit der Anwendung von Mathematik betrachtet. In den Bildungsstandards Mathematik sind zugehörige Kompetenzen im Bereich Mathematisch mo- 
dellieren gefasst (KMK 2003, 2012). Unter mathematischen Modellierungskompetenzen versteht man entsprechend die Fähigkeiten, Aufgaben mit Situationsbezug unter Einsatz mathematischer Modelle zu lösen (Klieme et al. 2001; KMK 2003, 2012). Dazu muss u. a. die Situation in ein mathematisches Modell übersetzt, innermathematisch bearbeitet und die mathematische Lösung vor dem Hintergrund der Situation interpretiert werden. Der prototypische Bearbeitungsprozess wird im sogenannten Modellierungskreislauf gefasst (z. B. Blum und Leiß 2005). Blomhøy und Jensen (2003) arbeiten heraus, dass die Bandbreite an Kontexten, die eine Person erfolgreich bewältigen kann, ein Qualitätsmerkmal für deren Mathematische Modellierungskompetenz ist (Merkmal Radius of action, dt. Reichweite). Auch hier wird angenommen, dass der Erwerb solcher breit anwendbarer Modellierungskompetenzen davon abhängt, ob die zugehörigen Lerngelegenheiten in ihren Kontexten variieren, die angezielte Bandbreite an Kontexten für den Radius of action also bereits beim Erwerb berücksichtigt wird.

Im berufsbildenden Bereich spricht Winther (2010) in Anlehnung an Anderson (1996) für Anforderungssituationen von einer kontextualen Färbung und nutzt das Aufgabenmerkmal der Spezifität im Sinne einer Kontextspezifität für die Klassifizierung, inwiefern eine Aufgabe berufliche Kontexte berücksichtigt (Winther und Klotz 2016): Eine (in der Berufsbildung auftretende) Aufgabe wird etwa als domänenverbunden mit niedriger Spezifität eingestuft, wenn allgemeine Rechen-, Leseund Schreibkompetenz zur Lösung ausreichen, und als domänenspezifisch, wenn sie sich ohne (kaufmännisches) Fachwissen und Können nicht lösen lässt. Diese domänenspezifischen Aufgaben sind dann deutlich kontextual gefärbt. Dabei ist anzumerken, dass eine Aufgabe auch in Hybridform vorliegen kann, wobei dann sowohl Anforderungen mit hoher und niedriger Spezifität vorkommen.

Damit ist deutlich geworden, dass der Unterschied zwischen den oben aufgezeigten Aufgabenbeispielen 1 und 2 bedeutsam für eine erfolgreiche oder nichterfolgreiche Bearbeitung sein kann. Obwohl sich beide Aufgaben auf allgemeinbildende mathematische Kompetenzen beziehen, ist die zweite kontextual gefärbt, indem sie einen Berufsfeldbezug aufweist. Die zweite Aufgabe kann damit als Anforderung im Sinne der berufsfeldbezogenen mathematischen Kompetenzen verstanden werden und deren Abgrenzbarkeit zu den allgemeinbildenden mathematischen Kompetenzen verdeutlichen. Auf Basis der kognitionspsychologischen Forschung ist anzunehmen, dass ein Transfer der erworbenen Kompetenzen zwischen zwei unterschiedlichen Anwendungskontexten nicht zwingend funktioniert. Der Radius of action für schulisch erworbene Kompetenzen würde damit berufsfeldbezogene mathematische Anforderungen eher nicht abdecken, wenn keine Lerngelegenheiten mit entsprechender kontextualer Färbung angeboten werden. Eine von uns durchgeführte Analyse von 18 allgemeinbildenden Mathematikschulbüchern der Klassenstufen 6-10 zeigt, dass darin kaum Lerngelegenheiten mit kaufmännischen Kontexten aus einer beruflichen Perspektiven vorkommen (von Hering et al. 2020). Nimmt man dieses Ergebnis als Indikator für die Kontexte der Lerngelegenheiten im aktuellen Mathematikunterricht, so würde daraus bei Auszubildenden derzeit eine beschränkte Nutzbarkeit ihrer allgemeinbildenden mathematischen Kompetenzen zur Bewältigung berufsfeldbezogener mathematischer Anforderungen folgen. 
Vor diesem Hintergrund könnte man erwarten, dass bei der Bearbeitung von allgemeinbildenden und berufsbezogenen Aufgaben Unterschiede in den kognitiven Bearbeitungsprozessen zu erkennen sind. Dies würde eine Abgrenzbarkeit von allgemeinbildenden mathematischen Kompetenzen von berufsfeldbezogenen mathematischen Kompetenzen stützen. Ob sich diese Unterscheidung qualitativ aufzeigen lässt, soll dabei entlang der folgenden Forschungsfragen gezeigt werden.

\subsection{Forschungsfragen}

Das übergreifende Erkenntnisinteresse der hier berichteten Studie lautet:

Lassen sich allgemeinbildende mathematische und berufsfeldbezogene mathematische Kompetenzen qualitativ voneinander abgrenzen?

Diese Frage konkretisiert sich im Rahmen der Studie auf folgende Weise:

1 Nutzen Industriekaufleute in der Ausbildung beim Bearbeiten berufsbezogener Mathematikaufgaben kognitive Prozesse, die als allgemeinbildend oder beruflich charakterisiert werden können, und wurden diese in der allgemeinbildenden Schule oder in der Ausbildung erlernt?

2 Welche Unterschiede/Gemeinsamkeiten sind für Industriekaufleute in der Ausbildung zwischen Parallelaufgaben, die auf allgemeinbildende mathematische oder berufsfeldbezogene mathematische Kompetenzen abzielen, salient?

Das Erkenntnisinteresse ergibt sich aus dem theoretischen Modell von Neumann et al. (2013) zu den mathematischen Kompetenzen am Übergang von der allgemeinbildenden Schule in die berufliche Erstausbildung. Die dort vorgeschlagenen berufsfeldbezogenen mathematischen Kompetenzen werden als Teilbereich der allgemeinbildenden mathematischen Kompetenzen angesehen, lassen sich aufgrund ihres beruflichen Kontextes aber theoretisch eingrenzen. Mittels quantitativer Methoden konnten allerdings Hinweise auf eine empirische Unterscheidung zweier entsprechender Tests gewonnen werden (Siebert und Heinze 2016). Eine weitere Untersuchung dieser Unterscheidung mit qualitativen Methoden auf Basis von Bearbeitungsprozessen wäre daher erstrebenswert.

Die beiden Forschungsfragen sollen mit Hilfe einer Mixed-Methods-Studie im querschnittlichen Design bearbeitet werden, die kognitive Prozesse bei Industriekaufleuten in der Ausbildung in unterschiedlichen (beruflichen und nicht-beruflichen) Anforderungen sichtbar macht. Dazu bearbeiten die Auszubildenden zuerst ein Set von Aufgaben, wobei die Hälfte der Aufgaben ohne Bezug zu kaufmännischen Kontexten steht und sich mit allgemeinbildenden mathematischen Kompetenzen ohne Berufsfeldbezug lösen lässt. Die andere Hälfte besteht aus mathematischstrukturell parallelen Aufgaben, die aber eine industriekaufmännische Perspektive einnehmen und so berufsfeldbezogene mathematische Kompetenzen erfordern. In einer anschließenden Stimulated-Recall-Interviewstudie werden Bearbeitungsprozesse expliziert, um auf genutzte Kompetenzen zu schließen. Dazu wird auch erhoben, ob 
Unterschiede und Gemeinsamkeiten in der Struktur der vorliegenden Parallelaufgaben wahrgenommen wurden.

Unter Berücksichtigung der kognitionspsychologischen Erkenntnisse erwarten wir in Bezug auf das übergeordnete Erkenntnisinteresse folgende Fälle:

1 Erkennen die Auszubildenden die Struktur der Parallelaufgaben nicht, so gehen wir davon aus, dass sie verschiedene Kompetenzen bei den Bearbeitungen genutzt haben. Wir werten dies entsprechend als Hinweis auf eine Abgrenzbarkeit von allgemeinbildenden mathematischen und berufsfeldbezogenen mathematischen Kompetenzen.

2 Erkennen die Auszubildenden die Struktur der Parallelaufgaben, bearbeiten die Aufgabenpaare aber trotzdem deutlich unterschiedlich und kongruent zu typischem schulischen bzw. beruflichen Vorgehen, so werten wir dies ebenfalls als Hinweis auf eine Abgrenzbarkeit der theoretischen Konstrukte.

3 Erkennen die Auszubildenden die Struktur der Parallelaufgaben und bearbeiten zudem die Aufgabenpaare auch auf die gleiche Art, dann werten wir dies (aufgrund des vorgängigen Erwerbs) als Anwendung allgemeinbildender mathematischer Kompetenzen. In diesem Fall könnten wir eine Abgrenzbarkeit der theoretischen Konstrukte nicht bestätigen.

\section{Methodisches Vorgehen}

\subsection{Design}

Es wurde eine Mixed-Methods-Studie im querschnittlichen Design in drei Schritten durchgeführt: Zunächst entwickelten wir Paare von kontextualisierten (Modellierungs-)Aufgaben (siehe Abschn. 3.2). Jedes Paar besteht aus einer Aufgabe mit einem beruflichen Kontext und einer Aufgabe mit einer isomorphen mathematischen Struktur in einem nicht-beruflichen Kontext. Beide Aufgaben können durch die Anwendung allgemeinbildender mathematischer Kompetenzen gelöst werden, die in der Sekundarstufe erworben wurden, unterscheiden sich aber in den (industrie-)kaufmännischen Anforderungen (vgl. Abschn. 2.3). Die Parallelität der Aufgabenpaare wurde in einem Expertenrating geprüft. Anschließend wurden diese Probleme von $N=42$ Auszubildenden als Test bearbeitet (siehe Abschn. 3.3). Diese Bearbeitungen wurden korrigiert und je ein Testwert für die Aufgaben mit und ohne beruflichen Bezug bestimmt. Auf Basis der Lösungsraten wurden dann $n=18$ Auszubildende der Stichprobe kriteriengeleitet für die vertiefte Analyse der kognitiven Prozesse ausgewählt und interviewt (siehe Abschn. 3.3 für Details). So konnte untersucht werden, ob sich innerhalb der parallelen Aufgaben gemäß des Erkenntnisinteresses Unterschiede in den Bearbeitungsprozessen nachweisen lassen. 


\subsection{Genutzte Aufgaben}

In der Studie sollten mathematische Modellierungsaufgaben genutzt werden, um berufliche oder nicht-berufliche allgemeinbildende mathematische Anforderungen zu transportieren (drei allgemeinbildende Aufgaben [A1-A3] und drei berufsbezogene Aufgaben [B1-B3], Aufgaben A1 \& A2, sowie B1 \& B2 im Anhang, Aufgaben A3 und B3 nachfolgend). Insbesondere die Vergleichbarkeit bzw. Parallelität jeweils zweier Aufgaben hinsichtlich der innermathematischen Struktur war dabei von zentraler Bedeutung, da nur so das weitere Vorgehen zur Beantwortung der Forschungsfragen sinnvoll durchgeführt werden konnte. Da ein solches Aufgabenset nicht vorliegt, wurden die Aufgaben für diesen Zweck entwickelt, wie im Folgenden detailliert dargestellt wird.

Als Ausgangspunkt für die berufsbezogenen Aufgaben diente der Aufgabenpool der Industrie- und Handelskammer für die Abschlussprüfungen der Industriekaufleute in der Ausbildung. Hier wurden drei Aufgaben ausgewählt, die aus mathematisch-inhaltlicher Sicht nicht die Anforderungen allgemeinbildender mathematischer Kompetenzen übersteigen, jedoch ggf. die Kenntnis einschlägiger Fachtermini voraussetzen. Dabei durfte das kaufmännische Fachwissen das Niveau des zweiten Ausbildungsjahres nicht überschreiten, um den Aufgabeneinsatz mit Auszubildenden am Ende des zweiten und dritten Lehrjahres sinnvoll durchführen zu können.

Abb. 2 zeigt eine solche Aufgabe (Aufgabe B3), wovon der erste Aufgabenteil folgend näher betrachtet wird. Für das Produkt Computertische liegen in B3.1 Informationen über die bei der Produktion anfallenden Kosten im Aufgabentext vor. In dieser Teilaufgabe soll der Angebotspreis berechnet werden. Für die Berechnung wird ein typisches (kaufmännisches) Schema (Zuschlagskalkulation) zugrunde gelegt. Dabei bilden die Herstellkosten sowohl die Zuschlagsgrundlage für den Verwaltungsgemeinkostenzuschlag als auch für den Vertriebsgemeinkostenzuschlag. Die Zuschlagsgrundlage für den Gewinn sind die Selbstkosten.

Als Grundlage für die folgende Entwicklung paralleler Aufgaben mit allgemeinbildenden mathematischen Anforderungen, wurden die ausgewählten Aufgaben zusätzlich gemäß des Kompetenzrasters der Bildungsstandards Mathematik (KMK 2003) analysiert. Die Anforderungsbereiche sind dabei immer in Relation zur Personengruppe zu setzen und berücksichtigen die Vertrautheit von Anforderungen.

Sie arbeiten in der Büromöbel HEIN KG und sind mit der Preiskalkulation für die neue Serie Computertische beauftragt. Pro Stück sind 200,00 € Herstellkosten angefallen. Darüber hinaus wird ein Verwaltungsgemeinkostenzuschlagssatz von $28 \%$ sowie ein Vertriebsgemeinkostenzuschlagssatz von $22 \%$ berücksichtigt.

1.) Wie hoch muss der Angebotspreis für einen Computertisch sein, wenn mit $12 \%$ Gewinn kalkuliert wird?

2.) Ein Kunde erteilt Ihnen einen Auftrag für Computertische und -stühle. Der komplette Angebotspreis liegt bei 448,00 € (inklusive $12 \%$ Gewinn). Dem Kunden werden $3 \%$ Skonto auf den Angebotspreis gewährt.

Wie hoch ist die daraus resultierende Gewinnabweichung in $€$ ?

Abb. 2 Genutzte berufsbezogene Aufgabe (Aufgabe B3) 
Für den Zweck dieser Studie wurde von Auszubildenden in der ersten Hälfte der beruflichen Erstausbildung ausgegangen.

Das Schema zur Berechnung des Angebotspreises beinhaltet bestimmte Kalküle, die für die korrekte Ermittlung des Angebotspreises unerlässlich sind, sodass die Aufgabe B3.1 der mathematischen Leitidee Zahl zuzuordnen ist. Weiter ist die allgemeine mathematische Kompetenz Mathematisch modellieren zentral. Die hier zugrunde liegende Modellierung erfolgt in mehreren Schritten: Zuerst müssen die Selbstkosten und dann kann erst der Gewinn berechnet werden. Es wird die Formel für den Prozentwert angewendet. Dabei muss keine umfangreiche Modellierung vorgenommen werden, da die Realsituation direkt in die Mathematik überführt werden kann. Da es sich um ein Standardmodell handelt und, wie im Folgenden zu sehen ist, ein typisches kaufmännisches Schema angewendet wird, ist die Aufgabe - für die hier angedachte Personengruppe - dem Anforderungsniveau Reproduzieren zuzuordnen.

Die Gemeinkostenzuschlagssätze werden in der Industriekaufleute-Ausbildung im ersten Lehrjahr im Zuge von Lernfeld 4 (Wertschöpfungsprozesse analysieren und beurteilen) im Rahmen der Kostenträgerstückrechnung behandelt (KMK 2002). Dazu wird die Zuschlagskalkulation als Angebotskalkulation durchgeführt. Das Ziel von Lernfeld 4 ist das Erfassen der beim betrieblichen Leistungsprozess entstehenden Kosten und Leistungen, die Berechnung und Beurteilung des kostenrechnerischen Wertschöpfungsbeitrags einzelner Produkte und des Betriebserfolgs (KMK 2002). Für die Lösung der Aufgabe ist kaufmännisches Vorwissen zum Begriff Angebotspreis und zum Umgang damit hilfreich. Die Kalkulation des Angebotspreises basiert auf einem typischen kaufmännischen Schema, das nicht unbedingt intuitiv ist, da der Gewinn auf die Selbstkosten berechnet wird, die anderen Zuschläge aber auf die Herstellkosten. Auch werden die beiden Zuschläge in diesem Schema in einem additiven Vorgehen parallel zueinander berechnet. Die Entscheidung zugunsten dieses Vorgehens und gegen eine multiplikative Nacheinander-Berechnung der Zuschläge muss auf Grundlage des kaufmännischen Vorwissens getroffen werden. Dennoch handelt es sich bei der Aufgabe um ein niedriges Anforderungsniveau, da mit dem angesprochenen Vorwissen durch die Anwendung eines Routineverfahrens nur Modellieren in eingeschränktem Umfang erforderlich ist. Die Spezifität der Aufgabe (nach Winther und Klotz 2016) ist hierbei als domänenverbunden, also auf einem (aus beruflicher Sicht) eher niedrigem Grad an beruflicher Färbung einzustufen. Dies begründet sich darin, dass die vorkommenden kaufmännisch-spezifischen Begriffe (Verwaltungsgemeinkostenzuschlagssatz, Vertriebsgemeinkostenzuschlagssatz) nicht zwingend bekannt sein müssen, um die Aufgabe erfolgreich zu bearbeiten. Die Aufgabe lässt sich auch durch allgemeine Rechen-, Lese- und Schreibkompetenz lösen. Dennoch bestimmen kaufmännische Fachtermini (z. B. „Herstellkosten“, „Skonto“, „Angebotspreis“) die Aufgabe und sorgen dafür, dass die Aufgabe den berufsfeldbezogenen mathematischen Kompetenzen aus dem Kompetenzmodell von Neumann et al. (2013) zuzuordnen ist.

Alle insgesamt sieben Teilaufgaben der drei berufsbezogenen Aufgaben wurden auf diese Weise anhand des Schemas zu den allgemeinbildenden mathematischen Kompetenzen aus den Bildungsstandards Mathematik (KMK 2003) einsortiert. Durch Neukontextualisierung entstanden anschließend je parallele Aufgaben, 
Im Bezirk der Arbeitsagentur Neuhausen sind ca. 20.000 Arbeitslose registriert. Zum 31.12.2014 droht die Schließung von zwei ortsansässigen Unternehmen. Laut offizieller Statistik würde das Unternehmen Stoff $\mathrm{GmbH}$ die Arbeitslosenzahl um $8 \%$ und das Unternehmen Reifen AG die Arbeitslosenzahl um $6 \%$ erhöhen.

1.) Nach dem die Unternehmen Stoff GmbH und Reifen AG geschlossen wurden, zeichnet sich im Jahr 2015 eine weitere allgemeine Erhöhung der Arbeitslosenquote um $4 \%$ ab. Wie viele Arbeitslose sind zum 31.12.2015 in Neuhausen registriert?

2.) Im benachbarten Bezirk der Arbeitsagentur Althausen hätte es zum 31.12.2015 ca. 44.800 Arbeitslose gegeben (inkl. der allgemeinen Steigerungsrate von $4 \%$ im Jahr 2015). Glücklicherweise konnten neue Unternehmen angesiedelt werden, die die befürchtete Arbeitslosenzahl um $3 \%$ reduziert haben. Wie hoch ist die Abweichung der tatsächlichen Arbeitslosenzahl von der befürchteten (in Personen)?

Abb. 3 Genutzte allgemeinbildende Aufgabe (Aufgabe A3)

die sich als möglichst wenig berufsspezifisch für das kaufmännische Feld erweisen sollen und in anderen gesellschaftlich relevanten Kontexten situiert wurden. Dabei wurde für die Parallelität innerhalb der drei Aufgabenpaare eine mathematische und strukturelle Äquivalenz angestrebt, wobei Unterschiede zwischen den Zahlenwerten der Aufgaben durch den Anspruch, möglichst realistische Aufgabe zu erhalten, begründet sind. Durch die Möglichkeit des Taschenrechnereinsatzes sollte dies jedoch nicht zu Unterschieden in der Aufgabenschwierigkeit führen.

Abb. 3 zeigt die allgemeinbildende Aufgabe A3, die durch Neukontextualisierung der berufsbezogenen Aufgabe B3 entstanden ist. In A3.1 soll die Arbeitslosenzahl einer Region nach Schließung zweier Arbeitgeber berechnet werden. Hierzu liegen alle nötigen Informationen im Aufgabentext vor. Die Berechnung erfolgt mathematisch äquivalent zur Berechnung des Angebotspreises in der berufsbezogenen Aufgabe B3.1 mit an den Aufgabenkontext angepassten realistischen Zahlenwerten. Die Berechnungsgrundlage für die Steigerung der Arbeitslosenzahlen nach SchlieBung der beiden Betriebe bildet jeweils die registrierte Arbeitslosenzahl, diejenige für die allgemeine Erhöhung 2015 die Arbeitslosenzahl Ende 2014 (nach Steigerung durch die Schließung der beiden Betriebe).

Die Zuordnung der Aufgabe zur mathematischen Leitidee Zahl und zur allgemeinen mathematischen Kompetenz Mathematisch modellieren lässt sich parallel zu Aufgabe B3.1 begründen. Auch hier sorgt die einfache Anwendung der Formel für den Prozentwert dafür, dass das Anforderungsniveau Reproduzieren für die angedachte Personengruppe nicht überschritten wird. Der alltagsnahe Kontext weist keinen kaufmännischen Berufsbezug und keine kaufmännischen Fachtermini auf. Die sprachliche Komplexität ist bei der allgemeinbildenden Aufgabe etwas geringer als bei der berufsbezogenen Aufgabe. Lange Fachwörter wie „Vertriebsgemeinkostenzuschlag" erfordern einen hohen Grad an Lesekompetenz. Demgegenüber steht ein kaufmännisches Schema, das zur Bearbeitung der Aufgabe B3.1 angewendet werden kann und so die Komplexität gegenüber Aufgabe A3.1 wieder angleicht, in der die erforderliche Lesekompetenz geringer ist, dafür aber kein entsprechendes Schema vorliegt. Auch in Aufgabe A3.1 sind die beiden Steigerungen der Arbeitslosenzahl unabhängig voneinander zu betrachten und somit additiv (und nicht nach- 
einander, also multiplikativ) der registrierten Arbeitslosenzahl zuzurechnen. Es wird vorausgesetzt, dass das Themenfeld der Arbeitslosigkeit als allgemein gesellschaftlich relevantes Thema bekannt ist und Begriffe wie Arbeitslosenzahl und Arbeitslosenquote kein spezifisches berufliches Wissen darstellen, sodass die Aufgabe im Modell von Neumann et al. (2013) den (nicht berufsspezifischen) allgemeinbildenden mathematischen Kompetenzen zuzuordnen ist. Insgesamt gesehen sind beide Aufgaben theoretisch mit allgemeinbildenden mathematischen Kompetenzen lösbar, wobei Aufgabe B3.1 aber den Teilbereich der berufsfeldbezogenen mathematischen Kompetenzen adressiert.

In einem mehrstufigen Experten-Konsens-Rating mit drei erfahrenen Mathematiklehrkräften sowie fünf Mathematikdidaktikerinnen und Mathematikdidaktikern wurden alle drei Aufgabenpaare (14 Teilaufgaben: zwei Aufgabenpaare mit je $2 \times 2$ Teilaufgaben und ein Aufgabenpaar mit $2 \times 3$ Teilaufgaben) gemäß des Kompetenzmodells der Bildungsstandards Mathematik (KMK 2003) eingeordnet, um die angezielte Parallelität zu prüfen. Wenn die parallele Aufgabenkonstruktion gelungen ist, dann sollten sich die Aufgaben in Bezug auf Leitideen, allgemeine mathematische Kompetenzen sowie Anforderungsbereiche nicht wesentlich unterscheiden. Es wäre aber plausibel, dass die Aufgaben mit beruflichen Anforderungen ggf. in Bezug auf den Anforderungsbereich höher eingeschätzt werden, weil dieser die Vertrautheit mit den abgebildeten Situationen berücksichtigt.

Tab. 1 zeigt die Ergebnisse dieses Experten-Konsens-Ratings. Die hier vorgestellten Aufgaben A3 und B3 wurden beispielsweise der allgemeinen mathematischen Kompetenz 3 (Mathematisch modellieren), der Leitidee 1 (Zahl) und den Anforderungsniveaus I (Aufgaben A3.1 \& B3.1) und II (Aufgaben A3.2 \& B3.2) zugeordnet. Fünf von sieben Teilaufgabenpaaren stimmen laut Rating in allen drei Dimensionen überein, die anderen beiden Teilaufgabenpaare unterscheiden sich lediglich geringfügig im Anforderungsniveau, wobei in allen Fällen erwartungsgemäß den berufsbezogenen Aufgaben das höhere Anforderungsniveau zugesprochen wurde. Auf diese

Tab. 1 Einstufung der verwendeten (Teil-)Aufgaben gemäß der Bildungsstandards Mathematik (allgemeinbildende Aufgaben: A1-A3, berufsbezogene Aufgaben: B1-B3)

\begin{tabular}{|c|c|c|c|}
\hline $\begin{array}{l}\text { (Teil-)Auf- } \\
\text { gabe }\end{array}$ & Allgemeine Kompetenz & Leitidee & Anforderungsniveau \\
\hline A1.1/B1.1 & $\begin{array}{l}\text { Mathematisch modellieren \& } \\
\text { Mathematische Darstellun- } \\
\text { gen verwenden }\end{array}$ & $\begin{array}{l}\text { Funktionaler } \\
\text { Zusammen- } \\
\text { hang }\end{array}$ & $\begin{array}{l}\text { I: Reproduzieren - II: Zusammenhänge } \\
\text { herstellen (A1.1) } \\
\text { II: Zusammenhänge herstellen (B1.1) }\end{array}$ \\
\hline $\mathrm{A} 1.2 / \mathrm{B} 1.2$ & $\begin{array}{l}\text { Mathematisch modellieren \& } \\
\text { Mathematische Darstellun- } \\
\text { gen verwenden }\end{array}$ & $\begin{array}{l}\text { Funktionaler } \\
\text { Zusammen- } \\
\text { hang }\end{array}$ & $\begin{array}{l}\text { I: Reproduzieren (A1.2) } \\
\text { II: Zusammenhänge herstellen (B1.2) }\end{array}$ \\
\hline $\mathrm{A} 1.3 / \mathrm{B} 1.3$ & $\begin{array}{l}\text { Mathematisch modellieren \& } \\
\text { Mathematische Darstellun- } \\
\text { gen verwenden }\end{array}$ & $\begin{array}{l}\text { Funktionaler } \\
\text { Zusammen- } \\
\text { hang }\end{array}$ & II: Zusammenhänge herstellen \\
\hline A2.1/B2.1 & Mathematisch modellieren & Zahl & II: Zusammenhänge herstellen \\
\hline $\mathrm{A} 2.2 / \mathrm{B} 2.2$ & Mathematisch modellieren & Zahl & I: Reproduzieren \\
\hline A3.1/B3.1 & Mathematisch modellieren & Zahl & I: Reproduzieren \\
\hline A $3.2 / B 3.2$ & Mathematisch modellieren & Zahl & II: Zusammenhänge herstellen \\
\hline
\end{tabular}

kursiv: Nicht-Übereinstimmung zwischen den parallel angedachten (Teil-)Aufgaben 
Weise konnte die strukturelle und mathematische Parallelität der Aufgaben bestätigt werden und wir gehen davon aus, dass die Entwicklung paralleler Aufgaben zum Zwecke der Untersuchung damit gelungen ist.

\subsection{Stichprobenauswahl und Aufgabeneinsatz}

Die so entwickelten Aufgaben wurden bei $N=42$ Industriekaufleuten in der Ausbildung (26 weiblich) eingesetzt. Davon befanden sich zum Zeitpunkt der Testung 18 am Ende des zweiten und 24 am Ende des dritten Lehrjahres. Beide Ausbildungsjahrgänge bildeten je eine vollständige Ausbildungsklasse für Industriekaufleute in einer Berufsschule. Das papierbasierte Testheft fragte in standardisierter Reihenfolge zunächst die berufsbezogenen Aufgaben B1-B3 und anschließend die allgemeinbildenden Aufgaben A1-A3 ab. Diese Anordnung wurde gewählt, da die Antwortprozesse bei berufsbezogenen Aufgaben im Fokus von Forschungsfrage 1 stehen und eine vorherige Bearbeitung der allgemeinbildenden Aufgaben diese Prozesse verzerren könnten. Die Auszubildenden hatten 70 min Bearbeitungszeit und konnten einen Taschenrechner als Hilfsmittel nutzen. Für jede Teilaufgabe war ausschließlich ein Zahlenwert als Lösung zugelassen, Notizen und Lösungswege wurden bei der Korrektur zunächst nicht beachtet. Für die Auswertung wurden die Lösungen der Teilaufgaben jeweils dichotom bewertet (1 Punkt pro korrekter Lösung). Anschließend wurden die Lösungsraten für jede Aufgabe bestimmt, wobei die unterschiedliche Anzahl an Teilaufgaben in der relativen Lösungsrate berücksichtigt wurde.

Für die Beantwortung der Forschungsfragen sind Unterschiede in Bezug auf Bearbeitungsprozesse auf individueller Ebene von Interesse, die in den schriftlichen Lösungen nicht sichtbar sind und deswegen in Interviews auf Basis einer Teilstichprobe zugänglich gemacht werden sollten. Für die Stimulated-Recall-Interviews wurde ein Verfahren der absichtsvollen Stichprobenziehung auf Basis der Lösungsraten angewendet (Przyborski und Wohlrab-Sahr 2014). Aus beiden Ausbildungsjahrgängen sollten jeweils drei Personen mit deutlich höherer Lösungsrate bei den berufsbezogenen Aufgaben als bei den allgemeinbildenden Aufgaben (mindestens drei berufsbezogene Teilaufgaben mehr korrekt gelöst als allgemeinbildende), drei Personen mit deutlich höherer Lösungsrate bei den allgemeinbildenden Aufgaben als bei den berufsbezogenen Aufgaben (mindestens drei allgemeinbildende Teilaufgaben mehr korrekt gelöst als berufsbezogene) und drei Personen mit hoher Übereinstimmung in der Lösungsrate bei beiden Aufgabenblöcken ausgewählt werden. Durch dieses Sampling-Verfahren ergeben sich 18 Auszubildende für die Interviews (jeweils neun pro Ausbildungsjahrgang), die ein heterogenes Bild bezüglich ihrer Lösungsraten abgeben und bei denen großes Potenzial vorhanden ist, dass in der qualitativen Interviewauswertung Indizien für eine Abgrenzbarkeit allgemeinbildender mathematischer und berufsfeldbezogener mathematischer Kompetenzen identifizierbar sind. 


\subsection{Leitfragengesteuerte Interviews}

\subsubsection{Durchführung der Stimulated-Recall-Interviews mithilfe eines Interviewleitfadens}

In den Interviews wurden zuerst die berufsbezogenen Aufgaben und die von den Auszubildenden erstellten Aufgabenbearbeitungen nach der Methode des Stimulated Recalls vorgelegt. Im Verlauf der Interviews wurden dann zusätzlich die allgemeinbildenden Aufgaben hinzugezogen. Die Interviews fanden mit sehr geringem zeitlichem Abstand zur Testdurchführung (1-3 Tage) statt und alle 18 durch das Verfahren der absichtsvollen Stichprobenziehung ausgewählten Auszubildenden konnten teilnehmen.

Auf Basis der Frageregeln nach Helfferich (2011) (z. B. offene und wertfreie Fragen formulieren, auf Oder- bzw. Mehrfachfragen verzichten) wurde ein teilstandardisierter Interviewleitfragenkatalog entwickelt. Die Antworten auf folgende Fragen bilden die Datenquelle für die Beantwortung der Forschungsfragen:

1. Beschreiben Sie Ihr Vorgehen zur Lösung der Teilaufgabe B [Aufgabenbezeichnung].

2. Gab es Schwierigkeiten beim Lösen der Aufgabe?

3. Welches Wissen haben Sie zur Lösung der Aufgabe genutzt?

4. Hat Ihnen zur Lösung der Aufgabe Vorwissen gefehlt?

5. Wo haben Sie diese Methoden gelernt?

6. Haben Sie diese Aufgaben schon einmal in der Schule oder im Betrieb gerechnet?

7. Welche Unterschiede/Gemeinsamkeiten sehen Sie zwischen den beiden Aufgaben B [Aufgabenbezeichnung] und A [Aufgabenbezeichnung]?

8. Welche Aufgabe fiel Ihnen leichter zu lösen?

Dabei verfolgt die erste, allgemeinere Frage den Ansatz der Stimulated-RecallMethode und wird mit Frage 2 und ggf. durch weitere gezielte Nachfragen konkretisiert. Die Fragen 3 und 4 gehen auf das angewendete (Vor-)Wissen der Auszubildenden ein und zielen auf die Art des Bearbeitungsprozesses ab, während insbesondere die Antworten auf die Fragen 5 und 6 Aufschluss über den Ort des Wissenserwerbs geben sollen (Forschungsfrage 1). Dabei wird im Interview der leichter verständliche Begriff Wissen verwendet, obgleich die Antwort als Indikator für kognitive Bearbeitungsprozesse der Auszubildenden genutzt wird. Die Fragen 7 und 8 befassen sich mit den Unterschieden und Gemeinsamkeiten der parallel angelegten Aufgabenpaare, umfassen also nicht nur die berufsbezogenen, sondern auch die allgemeinbildenden Aufgaben, und könnten damit Antworten auf Forschungsfrage 2 liefern.

\subsubsection{Kategoriensystem zum Bearbeitungsprozess und zum Ort des Wissenserwerbs (Forschungsfrage 1)}

Die Auswertung der Interviews geschieht auf Basis der Qualitativen Inhaltsanalyse nach Mayring (2015). Das entsprechende Kategoriensystem entstand deduktiv (Methode der Strukturierung, Mayring 2015) und kann dabei sowohl auf Antworten auf 
Tab. 2 Kategoriensystem für die kognitiven Bearbeitungsprozesse und den Ort des Wissenserwerbs

\begin{tabular}{|c|c|c|c|c|c|}
\hline \multicolumn{6}{|c|}{ Bearbeitungsprozess } \\
\hline Oberkategorien & \multicolumn{2}{|c|}{ Berufsspezifisch } & \multicolumn{2}{|c|}{ Allgemeinbildend } & Unbekannt \\
\hline Unterkategorien & Direkt & Indirekt & Direkt & Indirekt & - \\
\hline \multicolumn{6}{|c|}{ Ort des Wissenserwerbs } \\
\hline Oberkategorien & \multicolumn{2}{|c|}{ Berufsspezifisch } & \multicolumn{2}{|c|}{ Allgemeinbildend } & Unbekannt \\
\hline Unterkategorien & Direkt & Indirekt & Direkt & Indirekt & - \\
\hline
\end{tabular}

Tab. 3 Verwendete Kodierregeln für die Kategorie Bearbeitungsprozess

\begin{tabular}{llll}
\hline Ober-l Kriterien & Beispiel & \\
Unter- & & & \\
kategorie & & & \\
\hline
\end{tabular}

kategorie

Berufs- Zuordnung, wenn eindeutig gesagt spezifisch, wird, dass das in der Aufgabenbeardirekt beitung verwendete Schema aus einer berufsspezifischen Bildungseinrichtung bekannt ist. Dies kann auch in weiteren Erklärungen zur Lösungsstrategie erfolgen, die durch Zusatzfragen gegeben werden

Berufs- Zuordnung, wenn ein eindeutig berufsspezifisch, spezifischer Fachbegriff oder genaue indirekt Umschreibungen (Definitionen) für berufsspezifische Fachbegriffe verwendet werden, deren Herkunft jedoch nicht weiter erläutert wird

Allgemein- Zuordnung, wenn Rechenvorgänge wie bildend, Rechenoperationen angegeben werden, direkt die zusätzlich mit dem Hinweis auf eine allgemeine Bildungseinrichtung begründet werden. Hierzu dürfen Aussagen auf Nachfrage einbezogen werden, wenn z. B. gesagt wird, dass die Aufgabe rechnerisch gelöst wurde

Allgemein- Zuordnung, wenn mathematische Fachbildend, begriffe wie Differenz, Addition, Subindirekt traktion, Division, Funktionen, etc. angegeben werden, sodass aus dem Kontext hervorgeht, dass es sich um mathematische Argumentationen/ Gedankengänge (ohne berufsspezifischen Hintergrund) handelt

Unbekannt Zuordnung, wenn nur die Lösungswege oder Rechenoperationen allgemein beschrieben werden, sodass eine Zuordnung in eine der vier anderen Kategorien nicht möglich ist
P1: ,[...] hab ich eben die BreakEven-Point-Formel benutzt, die wir in Rechnungswesen hatten."

P4: ,,[...] BreakEven-Point ausgerechnet. Äh, und dazu hatten wir dann ja die Formel."

P6: „Hier hab ich, äh, die Differenz berechnet, also, die prozentuale Differenz, so wie ich das in der Schule früher gelernt hab."

P2: „Und dann habe ich mit dem Dreisatz oder, äh, mit Prozentrechnung den Wert ausgerechnet."

P8: „Ich hab die $200 €$ genommen und die $28 \%$ und die $22 \%$ draufgerechnet."
Das Unterrichtsfach

Rechnungswesen wird explizit im Zusammenhang mit dem Bearbeitungsprozess als Quelle für die Break-Even-PointFormel genannt

Der berufsspezifische (kaufmännische) Fachbegriff (BreakEven-Point) wird ohne Angabe der Herkunft genannt Als Vorgehensweise wird mathematisch begriffliches Arbeiten angegeben und dies wird auch explizit gemacht

Mathematische Fachbegriffe (ohne Berufsbezug) werden ohne direkten Verweis auf die allgemeinbildende Schule genannt

Der Lösungsweg wird allgemein beschrieben 
Tab. 4 Verwendete Kodierregeln für die Kategorie Ort des Wissenserwerbs

\begin{tabular}{|c|c|c|c|}
\hline $\begin{array}{l}\text { Ober-/ } \\
\text { Unter- } \\
\text { kategorie }\end{array}$ & Kriterien & Beispiel & Begründung \\
\hline $\begin{array}{l}\text { Berufs- } \\
\text { spezifisch, } \\
\text { direkt }\end{array}$ & $\begin{array}{l}\text { Zuordnung, wenn eindeutig gesagt wird, } \\
\text { dass die Wissensherkunft auf eine berufs- } \\
\text { spezifische Bildungseinrichtung zurückzu- } \\
\text { führen ist. Dazu zählen auch Beschreibung } \\
\text { wie „,in der Ausbildung“, „,in Rechnungs- } \\
\text { wesen“, ,,in ReWe“ oder ,,hier“. (Die } \\
\text { Interviews fanden in berufsschulischen } \\
\text { Räumen statt.) }\end{array}$ & $\begin{array}{l}\text { P7: „Das haben } \\
\text { wir jetzt in der } \\
\text { Berufsschule oft } \\
\text { genug durchge- } \\
\text { rechnet }[\ldots . . .\end{array}$ & $\begin{array}{l}\text { Die Berufsschule wird } \\
\text { explizit genannt }\end{array}$ \\
\hline $\begin{array}{l}\text { Berufs- } \\
\text { spezifisch, } \\
\text { indi- } \\
\text { rekt }\end{array}$ & $\begin{array}{l}\text { Zuordnung, wenn Umschreibungen für } \\
\text { berufsspezifische Fachbegriffe verwendet } \\
\text { werden }\end{array}$ & $\begin{array}{l}\text { P7: „Einmal das } \\
\text { Wissen, dass } \\
\text { Erlöse gleich } \\
\text { Kosten sein müs- } \\
\text { sen, damit sie } \\
\text { keinen Verlust } \\
\text { mehr machen } \\
{[\ldots]^{“}}\end{array}$ & $\begin{array}{l}\text { Die kaufmännischen } \\
\text { Begriffe Erlöse, Kosten } \\
\text { und Verlust deuten } \\
\text { auf Wissen aus einer } \\
\text { berufsspezifischen } \\
\text { Ausbildung }\end{array}$ \\
\hline $\begin{array}{l}\text { Allgemein- } \\
\text { bildend, } \\
\text { direkt }\end{array}$ & $\begin{array}{l}\text { Zuordnung, wenn eindeutig gesagt wird, } \\
\text { dass die Wissensherkunft eindeutig auf } \\
\text { eine allgemeine Bildungseinrichtung } \\
\text { zurückzuführen ist. Dazu zählen auch } \\
\text { Beschreibungen wie „Matheunterricht“, } \\
\text { „Grundwissen“, „Grundrechenarten“, } \\
\text { „Schulwissen“, oder „,früher in der Schu- } \\
\text { le“" }\end{array}$ & $\begin{array}{l}\text { P1: ,[...] aber } \\
\text { auch eben nor- } \\
\text { maler Mathema- } \\
\text { tikunterricht aus } \\
\text { - weiß ich nicht - } \\
\text { Realschule, sag } \\
\text { ich mal.“ }\end{array}$ & $\begin{array}{l}\text { Sowohl der Mathema- } \\
\text { tikunterricht als auch } \\
\text { die Realschule werden } \\
\text { explizit genannt }\end{array}$ \\
\hline $\begin{array}{l}\text { Allgemein- } \\
\text { bildend, } \\
\text { indi- } \\
\text { rekt }\end{array}$ & $\begin{array}{l}\text { Zuordnung, wenn mathematische Begriffe } \\
\text { oder Rechenoperationen (ohne berufsspe- } \\
\text { zifischen Hintergrund) verwendet werden } \\
\text { und evtl. die allgemeinbildende Schule als } \\
\text { Wissensgrundlage angedeutet wird }\end{array}$ & $\begin{array}{l}\text { P7: „Da hatte ich } \\
\text { mal gelernt, dass } \\
\text { man dann noch } \\
\text { kurz subtrahieren } \\
\text { muss und dann } \\
\text { war das fertig.“ }\end{array}$ & $\begin{array}{l}\text { Andeutung eines weiter } \\
\text { zurückliegenden Wis- } \\
\text { senserwerbs, der sich } \\
\text { auf Rechenoperationen } \\
\text { der allgemeinbildenden } \\
\text { Schule ohne berufli- } \\
\text { chen Kontext bezieht }\end{array}$ \\
\hline Unbekannt & $\begin{array}{l}\text { Zuordnung, wenn eine unbekannte bzw. } \\
\text { nicht eindeutig zuordenbare Wissensher- } \\
\text { kunft angegeben wird }\end{array}$ & $\begin{array}{l}\text { P7: „Keine Ah- } \\
\text { nung, ist mir } \\
\text { halt einfach so } \\
\text { im Hinterkopf } \\
\text { irgendwie. Ich } \\
\text { weiß nicht, wo } \\
\text { das herkommt.“ }\end{array}$ & $\begin{array}{l}\text { Es wird explizit ange- } \\
\text { geben, dass der Ort des } \\
\text { Wissenserwerbs hier } \\
\text { unbekannt ist }\end{array}$ \\
\hline
\end{tabular}

die Fragen nach dem kognitiven Bearbeitungsprozess (insbesondere Fragen 1-4), als auch auf Fragen nach dem Wissenserwerb (Fragen 5 und 6) angewendet werden. Dabei steht Ort des Wissenserwerbs hier verkürzend für den Ort des Erwerbs der den angewendeten kognitiven Bearbeitungsprozessen zugrundeliegenden Kompetenzen. Oberkategorien für die Klassifikation der Antworten sind dabei Berufsspezifischer, Allgemeinbildender und Unbekannter Bearbeitungsprozess sowie Berufsspezifischer, Allgemeinbildender und Unbekannter Ort des Wissenserwerbs (siehe Tab. 2). Zusätzlich existieren Oberkategorien für die Fragen 7 und 8 nach den Unterschieden und den Gemeinsamkeiten zwischen den parallel angelegten Aufgabenpaaren (siehe Abschn. 3.4.3). Teilweise ergaben sich die Unterkategorien direkt und indirekt, 
Tab. 5 Verwendete Kodierregeln zu den wahrgenommenen Unterschieden und Gemeinsamkeiten der parallel angelegten Aufgabenpaare

\begin{tabular}{|c|c|c|c|}
\hline $\begin{array}{l}\text { Ober-/ } \\
\text { Unter- } \\
\text { kategorie }\end{array}$ & Kriterien & Beispiel & Begründung \\
\hline $\begin{array}{l}\text { Mathe- } \\
\text { matische } \\
\text { Struktur, } \\
\text { Gemein- } \\
\text { samkei- } \\
\text { ten }\end{array}$ & $\begin{array}{l}\text { Zuordnung, wenn iden- } \\
\text { tische Lösungswege an- } \\
\text { gegeben bzw. die Aufga- } \\
\text { benstellungen verglichen } \\
\text { und dabei die rechneri- } \\
\text { schen Gemeinsamkeiten } \\
\text { herausgestellt werden }\end{array}$ & $\begin{array}{l}\text { P3: „[...] und der Lösungsweg war für } \\
\text { mich relativ gleich.“ }\end{array}$ & $\begin{array}{l}\text { Die Gleichheit } \\
\text { bzw. Ähnlichkeit } \\
\text { der Lösungs- } \\
\text { wege beider } \\
\text { Aufgaben wird } \\
\text { explizit genannt }\end{array}$ \\
\hline $\begin{array}{l}\text { Mathe- } \\
\text { matische } \\
\text { Struktur, } \\
\text { Unter- } \\
\text { schiede }\end{array}$ & $\begin{array}{l}\text { Zuordnung, wenn verschie- } \\
\text { dene Lösungswege be- } \\
\text { schrieben werden oder die } \\
\text { Auszubildenden eindeutig } \\
\text { rechnerische Unterschiede } \\
\text { angeben }\end{array}$ & $\begin{array}{l}\text { P11: „Hier war für mich der Un- } \\
\text { terschied, dass ich den ersten Wert } \\
\text { ja schon hatte, also die } 44.800 \text {, die } \\
\text { waren ja vorgegeben. Ähm, und } \\
\text { das hatte ich im zweiten ja nicht, da } \\
\text { musste ich den Gewinn ja erst noch } \\
\text { ausrechnen.“ }\end{array}$ & $\begin{array}{l}\text { Unterschiede } \\
\text { zwischen den } \\
\text { Lösungswegen } \\
\text { beider Aufgaben } \\
\text { werden explizit } \\
\text { genannt }\end{array}$ \\
\hline $\begin{array}{l}\text { Oberflächen- } \\
\text { merkmale, } \\
\text { Gemein- } \\
\text { samkei- } \\
\text { ten }\end{array}$ & $\begin{array}{l}\text { Zuordnung, wenn Ge- } \\
\text { meinsamkeiten auf der } \\
\text { Sichtstruktur angegeben } \\
\text { werden }\end{array}$ & $\begin{array}{l}\text { P7: „,...] Tabellen schon mal ähnlich } \\
\text { aufgebaut“ }\end{array}$ & $\begin{array}{l}\text { Die angegebene } \\
\text { Gemeinsamkeit } \\
\text { ist oberflächlich } \\
\text { ohne inhaltliche } \\
\text { (mathematische) } \\
\text { Füllung }\end{array}$ \\
\hline $\begin{array}{l}\text { Oberflächen- } \\
\text { merkmale, } \\
\text { Unter- } \\
\text { schiede }\end{array}$ & $\begin{array}{l}\text { Zuordnung, wenn Unter- } \\
\text { schiede auf der Sichtstruk- } \\
\text { tur angegeben werden }\end{array}$ & $\begin{array}{l}\text { P5: „Die Abbildungen sehen schon } \\
\text { sehr unterschiedlich aus.“ }\end{array}$ & $\begin{array}{l}\text { Der angegebe- } \\
\text { ne Unterschied } \\
\text { beschreibt ein } \\
\text { Oberflächen- } \\
\text { merkmal }\end{array}$ \\
\hline
\end{tabular}

die festhalten, inwiefern die Kategorisierung in den Aussagen direkt erkennbar war oder aber eine Inferenz erforderte. Im Folgenden werden hierzu Kodierregeln und authentische Beispiele exemplarisch vorgestellt.

Tab. 3 stellt die verwendeten Kodierregeln für die Kategorie Bearbeitungsprozess vor. Eine Aussage zum Bearbeitungsprozess wird beispielsweise der berufsspezifischen Oberkategorie in der direkten Ausprägung zugeordnet, wenn eindeutig gesagt wird, dass das in der Aufgabenbearbeitung verwendete Schema aus einer berufsspezifischen Bildungseinrichtung bekannt ist. Dies kann auch in weiteren Erklärungen zur Lösungsstrategie erfolgen, die durch Zusatzfragen gegeben werden.

Parallel dazu beschreibt Tab. 4 die verwendeten Kodierregeln für die Kategorie Ort des Wissenserwerbs.

Eine Aussage zum Ort des Wissenserwerbs wird zum Beispiel der allgemeinbildenden Oberkategorie in der direkten Ausprägung zugeordnet, wenn gesagt wird, dass die Wissensherkunft eindeutig auf eine allgemeine Bildungseinrichtung zurückzuführen ist. Dazu zählen auch Beschreibungen wie „Matheunterricht“, „Grundwissen“, „Grundrechenarten“, „Schulwissen“, oder „, früher in der Schule“.

Für jedes der 18 durchgeführten Interviews und jede der sieben berufsbezogenen Teilaufgaben soll auf diese Weise sowohl der Bearbeitungsprozess als auch der Ort des Wissenserwerbs klassifiziert werden, sodass eine Gesamtheit von 126 Ana- 
Tab. 6 Mittelwert und Standardabweichung der Lösungsraten für die allgemeinbildenden Aufgaben (A) und berufsbezogenen Aufgaben (B) aufgeteilt nach Lehrjahr der Auszubildenden $\left(N_{2}=18\right.$ im zweiten Ausbildungsjahr, $N_{3}=24$ im dritten Ausbildungsjahr) und kumuliert $(N=42)$

\begin{tabular}{lllllll}
\hline $\mathrm{M}(S D)$ & 2. Lehrjahr & & 3. Lehrjahr & Gesamt & \\
& $\mathrm{A}$ & $\mathrm{B}$ & $\mathrm{A}$ & $\mathrm{B}$ & $\mathrm{A}$ & $\mathrm{B}$ \\
\hline Aufgaben & 0,65 & 0,74 & 0,53 & 0,68 & 0,58 & 0,71 \\
A1 \& B1 & $(0,33)$ & $(0,28)$ & $(0,28)$ & $(0,21)$ & $(0,30)$ & $(0,24)$ \\
Aufgaben & 0,54 & 0,47 & 0,49 & 0,39 & 0,51 & 0,42 \\
A2 \& B2 & $(0,40)$ & $(0,23)$ & $(0,36)$ & $(0,27)$ & $(0,37)$ & $(0,25)$ \\
Aufgaben & 0,69 & 0,56 & 0,56 & 0,63 & 0,62 & 0,60 \\
A3 \& B3 & $(0,35)$ & $(0,38)$ & $(0,37)$ & $(0,34)$ & $(0,36)$ & $(0,35)$ \\
Gesamt & 0,61 & 0,58 & 0,52 & 0,54 & 0,56 & 0,56 \\
$(N=18 / 24 / 42)$ & $(0,29)$ & $(0,17)$ & $(0,22)$ & $(0,17)$ & $(0,25)$ & $(0,17)$ \\
\hline
\end{tabular}

lyseeinheiten zum Bearbeitungsprozess sowie 126 Analyseeinheiten zum Ort des Wissenserwerbs betrachtet und kodiert wird.

Eine Bearbeitung werten wir für die Beantwortung der Forschungsfrage 1 als berufsspezifisch, wenn mindestens eine der beiden zusammengehörigen Analyseeinheiten (Bearbeitungsprozess und Ort des Wissenserwerbs für dieselbe Teilaufgabe im selben Interview) in die berufsspezifische Oberkategorie (direkt oder indirekt) fällt. Für diese Einteilung als berufsspezifische Bearbeitung auch bei einer von zwei berufsspezifischen Ausprägungen spielt die Chronologie der Bildungseinrichtungen im Verlauf der individuellen Bildungskarriere (zunächst allgemeinbildender Unterricht, anschließend berufliche Bildung) die entscheidende Rolle: Berufliche mathematische Bearbeitungsprozesse werden auf der fachlichen Grundlage der allgemeinbildenden mathematischen Prozesse durchlaufen, weswegen wir gemischte Angaben im Stimulated Recall als (zumindest teilweise) basierend auf beruflichen Prozessen werten.

\subsubsection{Kategoriensystem zu den Unterschiedenen und Gemeinsamkeiten der parallel angelegten Aufgabenpaare (Forschungsfrage 2)}

Auch die Antworten auf die Fragen nach den wahrgenommenen Gemeinsamkeiten und Unterschieden zwischen den parallel angelegten Aufgabenpaaren wurden mittels eines Kategoriensystems analysiert. Die Oberkategorien sind dabei Mathematische Struktur und Oberflächenmerkmale. Als Unterkategorien fungieren hierbei jeweils die Unterschiede sowie die Gemeinsamkeiten der mathematischen Struktur und der Oberflächenmerkmale.

Tab. 5 zeigt die verwendeten Kodierregeln zu den wahrgenommenen Unterschieden und Gemeinsamkeiten der parallel angelegten Aufgabenpaare. Eine Aussage wird beispielsweise als Gemeinsamkeit in der mathematischen Struktur kodiert, wenn identische Lösungswege angegeben bzw. die Aufgabenstellungen verglichen und dabei die rechnerischen Gemeinsamkeiten herausgestellt werden.

Für jedes der 18 durchgeführten Interviews und jedes der drei parallel angelegten Aufgabenpaare sollen auf diese Weise die wahrgenommenen Gemeinsamkeiten und Unterschiede sowohl in Bezug auf die mathematische Struktur als auch die Oberflächenmerkmale der Aufgaben klassifiziert werden, sodass eine Gesamtheit 
Tab. 7 Prozentuale Aufteilung der Bearbeitungen in die Kategorien allgemeinbildend, berufsspezifisch und unbekannt in der Gesamtstichprobe, aufgeteilt nach Lehrjahren sowie aufgeteilt nach Sampling-Gruppen

\begin{tabular}{llll}
\hline Klassifizierung der Bearbeitung & $\begin{array}{l}\text { Allgemein- } \\
\text { bildend } \\
\text { (in \%) }\end{array}$ & $\begin{array}{l}\text { Berufsspezifisch } \\
\text { (in \%) }\end{array}$ & $\begin{array}{l}\text { Unbekannt } \\
\text { (in \%) }\end{array}$ \\
\hline $\begin{array}{l}\text { Gesamt } \\
\text { (18 Interviews, 126 Analyseeinheiten) }\end{array}$ & 25 & 67 & 7 \\
$\begin{array}{l}\text { 2. Lehrjahr } \\
\text { (9 Interviews, 63 Analyseeinheiten) }\end{array}$ & 21 & 73 & 8 \\
$\begin{array}{l}\text { 3. Lehrjahr } \\
\text { (9 Interviews, 63 Analyseeinheiten) }\end{array}$ & 30 & 62 & 7 \\
$\begin{array}{l}\text { Sampling-Gruppe mit höherer Lösungsrate bei den } \\
\text { allgemeinbildenden Aufgaben } \\
\text { (6 Interviews, 42 Analyseeinheiten) }\end{array}$ & 29 & 64 & 5 \\
$\begin{array}{l}\text { Sampling-Gruppe mit höherer Lösungsrate bei den } \\
\text { berufsspezifischen Aufgaben } \\
\text { (6 Interviews, 42 Analyseeinheiten) }\end{array}$ & 19 & 76 & 10 \\
$\begin{array}{l}\text { Sampling-Gruppe mit ähnlicher Lösungsrate bei } \\
\text { allgemeinbildenden und berufsspezifischen Aufgaben } \\
\text { (6 Interviews, 42 Analyseeinheiten) }\end{array}$ & 29 & 62 & \\
\hline
\end{tabular}

von 54 Analyseeinheiten zur mathematischen Struktur und 54 Analyseeinheiten zu den Oberflächenmerkmalen betrachtet und kodiert wird, um Forschungsfrage 2 zu beantworten.

\subsubsection{Qualität des Kategoriensystems}

Zur Bestimmung der Objektivität des dargestellten Analyse-Verfahrens wurden 9 der 18 Interviews und damit insgesamt 180 von 360 Analyseeinheiten $(50 \%)$ unabhängig zweitkodiert. Dabei ergaben sich hohe Werte der Interrater-Reliabilität von $p_{0}=0,91$ (prozentuale Übereinstimmung), $\kappa=0,89$ (Fleiss' $\kappa$ ), die deutlich über dem üblichen Cut-Off-Wert von 0,75 liegen (Fleiss et al. 2003).

\section{Ergebnisse}

Für die den Interviews vorangestellten Testaufgaben zeigt Tab. 6 die deskriptiven Statistiken der Ergebnisse auf Aufgabenebene und für die Aufgabentypen. Bezogen auf die Gesamtstichprobe ergibt ein t-Test für die Unterschiede in den Lösungsraten innerhalb der Aufgabenpaare nur zwischen Aufgabe A1 und B1 einen signifikanten Unterschied $(t(40)=2,58 ; d=0,44 ; p=0,014)$, wobei die berufliche Aufgabe besser gelöst wurde. Im Expertenrating wurde ebenfalls nur für dieses Aufgabenpaar ein Unterschied vermutet, allerdings wurde hier umgekehrt der allgemeinbildenden Aufgabe ein leicht geringeres Anforderungsniveau attestiert (Tab. 1). Der Unterschied erwies sich in den Subgruppen des 2. bzw. 3. Lehrjahrs jeweils als nicht signifikant.

In beiden Lehrjahren gab es gemäß der angelegten Kriterien für die drei Sampling-Kategorien (höhere Lösungsrate bei den berufsbezogenen Aufgaben, höhere Lö- 
sungsrate bei den allgemeinbildenden Aufgaben, hohe Übereinstimmung zwischen den Lösungsraten) jeweils drei Personen, die für die anschließenden Interviews ausgewählt wurden und auch daran teilnahmen.

\subsection{Ergebnisse zum Bearbeitungsprozess und zum Ort des Wissenserwerbs (Forschungsfrage 1)}

Von den insgesamt 126 analysierten Bearbeitungen berufsbezogener Teilaufgaben (18 Interviews mit 7 Teilaufgaben) fielen $67 \%$ in die Kategorie des berufsspezifischen Bearbeitungsprozesses (direkt oder indirekt) und/oder in die Kategorie des berufsspezifischen Orts des Wissenserwerbs (direkt oder indirekt). Wir gehen also davon aus, dass diese berufsspezifisch bearbeitet wurden ${ }^{3}$. Schränkt man die Untersuchung auf erfolgreich gelöste Teilaufgaben ein (da eine nicht vorhandene Kompetenz nicht berufsspezifisch sein kann), ergibt sich ein Anteil an berufsspezifischen Bearbeitungen von $70 \%$.

Tab. 7 zeigt die prozentuale Aufteilung der Bearbeitungen in die Kategorien allgemeinbildend, berufsspezifisch und unbekannt in der Gesamtstichprobe, aufgeteilt nach Lehrjahren sowie aufgeteilt nach Sampling-Gruppen. Dabei zeigen sich weder zwischen den drei unterschiedlichen Sampling-Gruppen $\left(\chi^{2}(d f=4)=2,40 ; p=0,70\right)$ noch zwischen beiden Ausbildungsjahrgängen $\left(\chi^{2}(d f=2)=1,81 ; p=0,41\right)$ signifikante Unterschiede. Dabei ist zu betonen, dass in allen Substichproben der Anteil der Bearbeitungen, die als berufsspezifisch klassifiziert wurden, bei mindestens $62 \%$ liegt, die berufsbezogenen Aufgaben also durchweg häufiger berufsspezifische denn allgemeine mathematische Bearbeitungsprozesse hervorgerufen haben. Dies liefert die Antwort auf die erste Forschungsfrage.

\subsection{Ergebnisse zu den Unterschieden und Gemeinsamkeiten der parallel angelegten Aufgabenpaare (Forschungsfrage 2)}

Die vorliegenden 54 Aufgabenvergleiche (18 Interviews, je 3 Aufgabenpaare) wurden gemäß des Kategoriensystems aus Abschn. 3.4.3 kodiert. In 65\% der Fälle nennen die Auszubildenden sowohl Gemeinsamkeiten in der mathematischen Struktur als auch Unterschiede bezüglich der Oberflächenmerkmale der Aufgabenpaare, erkennen also das intendierte Setting im Aufgabendesign. Betrachtet man die beiden Oberkategorien separat sind es $76 \%$ (in 41 von 54 Fällen werden Unterschiede bezüglich der Oberflächenmerkmale angegeben) respektive $85 \%$ (in 46 von 54 Fällen werden Gemeinsamkeiten in der mathematischen Struktur angegeben).

Für die meisten Industriekaufleute in der Ausbildung sind also zwischen den Parallelaufgaben, die auf allgemeinbildende mathematische und berufsfeldbezogene mathematische Kompetenzen abzielen, die Unterschiede bezüglich der Oberflächen-

\footnotetext{
3 Wie oben dargelegt, hatten wir mit der Chronologie der mathematischen Ausbildung begründet, dass es für die Klassifizierung einer Bearbeitung als berufsspezifisch in dieser Studie genügte, dass Bearbeitungsprozess oder Ort des Wissenserwerbs als berufsspezifisch erkennbar waren. Wären diese Fälle strenger als allgemeinbildende Bearbeitung klassifiziert worden, so ergäbe sich noch immer ein Anteil von $56 \%$ an berufsspezifischen Bearbeitungen insgesamt.
} 
merkmale und die Gemeinsamkeiten in der mathematischen Struktur salient. Dies liefert die Antwort auf die zweite Forschungsfrage.

Die Auszubildenden erkennen also die Struktur der Parallelaufgaben. Da sie innerhalb der Aufgabenpaare zudem mehrheitlich unterschiedliche kognitive Bearbeitungsprozesse unterschiedlicher Herkunft erkennen lassen, tritt mit Blick auf die Kombination der Forschungsfragen 1 und 2 der zweite der drei möglichen vorab aufgestellten Fälle zur Beantwortung des übergeordneten Erkenntnisinteresses ein (vgl. Abschn. 2.4). In diesem Sinne bestätigt sich die empirische Abgrenzbarkeit der allgemeinbildenden und berufsfeldbezogenen mathematischen Kompetenzen.

\section{Fallbasierte Illustration der Ergebnisse}

Nach der qualitativen Inhaltsanalyse und ihrer summativen Auswertung sollen die Ergebnisse anhand eines Falles illustriert werden. Im folgenden Interviewausschnitt wird zunächst nach Bearbeitungsprozess und Ort des Wissenserwerbs bei der Beantwortung der berufsbezogenen Teilaufgabe B2.1 gefragt.

I: Welches Wissen haben Sie dafür genutzt?

P5: Also sowas hier machen wir jetzt grade sogar in. ... Hm. In GP1 machen wir das gerade.

I: Also Berufsschulwissen?

P5: Genau. Aber das könnt ich, denk ich mal, auch ... ja, mit Realschulwissen, denk ich mal, könnte man das auch hinbekommen.

Dabei liefert P5 mit GP1 (Unterrichtsfach Geschäftsprozesse) eine direkte Angabe eines berufsspezifischen Orts des Wissenserwerbs, sodass hier von einer Anwendung berufsspezifischen Wissens bei der Aufgabenbearbeitung durch P5 ausgegangen werden kann. Interessanterweise wird in der nächsten Aussage (,,ja, mit Realschulwissen, denk ich mal, könnte man das auch hinbekommen. ") der Kern der hier behandelten Forschungsfrage indirekt aufgegriffen. Berufsfeldbezogene mathematische Kompetenzen können als Teil der allgemeinbildenden mathematischen Kompetenzen angesehen werden, aber lassen sich möglicherweise auch von allgemeinbildenden mathematischen Kompetenzen abgrenzen. Praktisch interessiert also genau die Frage, ob man es ,,auch hinbekommt“, wenn man reines „Realschulwissen" (im Sinne von allgemeinbildenden mathematischen Kompetenzen) anwendet.

P5 konnte dabei die berufsbezogene Teilaufgabe B2.1 lösen, die parallel angedachte allgemeinbildende Teilaufgabe A2.1 jedoch nicht. Die folgende Passage deutet an, dass P5 dabei sowohl die Unterschiede bezüglich der Oberflächenmerkmale als auch die Gemeinsamkeiten in der mathematischen Struktur erkannt hat:

P5: Die [gemeint sind Teilaufgaben] ähneln sich hier ja auch wieder.

I: Ja, und welche Gemeinsamkeiten sehen Sie zwischen den beiden Aufgaben?

P5: Ja an sich ist es ja auch wieder das Gleiche. Ähm. Einmal wird halt ausgerechnet, welcher, äh, Lieferant der günstigste ist. Und bei dem Diätplan wird ausgerechnet, welcher Diätplan halt für ihn am sinnvollsten 
wär'. Also, das hat halt nichts mit Geld zu tun. (Pause) Also, einmal halt was Kaufmännisches und einmal halt was Nicht-Kaufmännisches.

I: $\quad$ Ähm, wenn Sie sich Ihre Rechnung angucken, also, sehen Sie da Unterschiede bei der Rechnung?

P5: Also der erste Schritt ist auf jeden Fall schon mal genau gleich. Hier habe ich ja den Preis mal die Menge gerechnet und hier habe ich das Gewicht mal die, den Tagesbedarf ausgerechnet [...]

P5 sieht also die angelegten Unterschiede bezüglich der Oberflächenmerkmale und die Gemeinsamkeiten in der mathematischen Struktur. Bei der Bearbeitung der Aufgaben lagen alle Teilaufgaben in Form eines Testhefts gesammelt vor und der Einsatz eines Taschenrechners war gestattet, um gegebenenfalls Probleme, die durch unterschiedliche Zahlenwerte entstehen, zu umgehen. Trotzdem konnte P5 die berufsbezogene Teilaufgabe lösen und die allgemeinbildende Teilaufgabe nicht. In der folgenden Passage wird P5 darauf angesprochen:

I: Okay, gut. Welche Aufgabe fiel Ihnen leichter zu lösen?

P5: Also, ich fand die kaufmännische Aufgabe auch wieder ein bisschen angenehmer. Auch, weil man damit, denk' ich mal, eher zu tun hat als mit sowas [allgemeinbildende Teilaufgabe] hier.

I: Okay, vielen Dank.

P5: Also, ich fand jetzt nicht unbedingt, dass eine von den beiden schwerer war, aber angenehmer fand ich auf jeden Fall die kaufmännische Aufgabe.

I: Okay. Also von der Rechnung her fanden Sie es nicht schwieriger, sondern eher, dass der Kontext für Sie ansprechender war, oder was?

P5: Genau.

In diesen Passagen lässt sich ein Großteil des von uns angelegten Settings wiederfinden. Die Aufgabenpaare wurden hinsichtlich der verwendeten allgemeinbildenden mathematischen Kompetenzen auf ihre fachliche Äquivalenz bzw. Parallelität geprüft, was bestätigt werden konnte, und auch P5 ,fand jetzt nicht unbedingt, dass eine von den beiden schwerer war“. Für die Lösung der berufsbezogenen Aufgabe nutzte P5 berufsspezifische kognitive Prozesse, was P5 näher liegt: „Auch, weil man damit, denk ich mal, eher zu tun hat als mit sowas hier." Neben den angedachten und durch P5 identifizierten Gemeinsamkeiten und Unterschieden zwischen den Aufgabenpaaren wurden die unterschiedlichen Kontexte unterschiedlich aufgenommen. Anknüpfend an die kognitionspsychologische Forschung zeigt sich, dass die Unterschiede im Kontext für diese Person entscheidend sind. Dies zeigt sich auch darin, dass P5 eine (die berufsbezogene) Aufgabe lösen kann und die andere (die allgemeinbildende) nicht.

Das hier gezeigte Interview illustrieren dabei exemplarisch den im Zuge der Forschungsfragen aufgestellten zweiten Fall (Abschn. 2.4), der in der hier berichteten Studie mehrheitlich zutraf. Die Struktur der Parallelaufgaben wird von den Auszubildenden wahrgenommen, die beiden Aufgaben aber dennoch unterschiedlich bearbeitet. So ergibt sich ein valides Argument für die Stützung der angenommenen 
Abgrenzbarkeit von allgemeinbildenden mathematischen und berufsfeldbezogenen mathematischen Kompetenzen mittels eines qualitativen Zugangs.

\section{Zusammenfassung, Diskussion \& Ausblick}

Studien zu Kompetenzverläufen im Übergang von der allgemeinbildenden Schule in die berufliche Erstausbildung stehen vor der Herausforderung, dass in den beiden Bildungsphasen unterschiedliche Kompetenzbegriffe zugrunde gelegt werden. Das von Neumann et al. (2013) vorgeschlagene Konstrukt der berufsfeldbezogenen Kompetenzen könnte eine Verbindung darstellen. Angewendet für den Bereich Mathematik und mathematikreiche Berufe erschienen dann berufsfeldbezogene mathematische Kompetenzen als potenzielle Mittler zwischen allgemeinbildenden mathematischen Kompetenzen und beruflichen Kompetenzen (vgl. Abschn. 2.2). Sie sind auf Grundlage von allgemeinbildenden mathematischen Kompetenzen definiert, aber stets mit der Anwendung in einem berufsspezifischen Kontext verknüpft. Wie in Abschn. 2.3 mit Bezug zu unterschiedlichen Theorien dargestellt, ist es möglich, dass sich neben der theoretischen Eingrenzung sogar eine empirische Abgrenzbarkeit von allgemeinbildenden mathematischen und berufsfeldbezogenen mathematischen Kompetenzen stützen lässt. Auf eine solche Abgrenzbarkeit deuten bereits erste statistische Analysen auf Basis von Testwerten hin. Ob sich diese Unterscheidung auch qualitativ in kognitiven Bearbeitungsprozessen abbilden lässt, war bislang offen und sollte in dieser Studie am Beispiel eines kaufmännischen Berufs untersucht werden.

Die vorliegende Studie setzte bei $N=42$ Industriekaufleuten in der Ausbildung in einem Parallel-Design Aufgaben ein, die berufsfeldbezogene mathematische Kompetenzen und allgemeinbildende mathematische Kompetenzen adressieren. Mit einer kriteriengeleitet ausgewählten Teilstichprobe wurden 18 Stimulated-Recall-Interviews u. a. zum Bearbeitungsprozess, zum Ort des Wissenserwerbs und zur Parallelität der genutzten Aufgaben geführt und diese mittels der Qualitativen Inhaltsanalyse nach Mayring (2015) ausgewertet. Es konnte gezeigt werden, dass die Auszubildenden die Mathematikaufgaben im beruflichen Kontext mehrheitlich unter einer berufsspezifischen Sichtweise lösen und etwa auf berufsspezifisches Wissen zurückgreifen. Die angedachten Unterschiede bezüglich der Oberflächenmerkmale und Gemeinsamkeiten in der mathematischen Struktur zwischen den Aufgabenpaaren wurden von den Auszubildenden dabei mehrheitlich erkannt. Trotzdem kam es bei den einzelnen Auszubildenden zu unterschiedlichen Problemen und Erfolgen bei der Bearbeitung der Aufgabenpaare mit beruflichem und nicht-beruflichem Kontext. Insgesamt stützen die qualitativen Ergebnisse die theoretische Abgrenzung der allgemeinbildenden mathematischen und der berufsfeldbezogenen mathematischen Kompetenzen.

Betrachtet man die bisherigen quantitativen und die hier berichteten qualitativen Ergebnisse, so bietet das Konstrukt der berufsfeldbezogenen mathematischen Kompetenzen eine Möglichkeit der Modellierung mathematischer Kompetenzen im Übergang von der allgemeinbildenden Schule in die berufliche Ausbildung. Es ergänzt damit die Modellierung der Kompetenzen aus der Perspektive der Berufsforschung (Winther et al. 2013) um die Forschungsperspektive der schulfachbezogenen 
Bildungsforschung und erlaubt dabei insbesondere eine Anschlussfähigkeit an die mathematikdidaktische Forschung im Sekundarbereich. Zugänge dieser Art sind beispielsweise von Bedeutung im Zusammenhang mit Fragen zur Berufsorientierung im allgemeinbildenden Mathematikunterricht. Aktuelle Veränderungen im Verhältnis von beruflicher und akademischer Ausbildung sowie ein gesellschaftlicher Strukturwandel hin zur Wissens- und Dienstleistungsgesellschaft stellen das berufliche Ausbildungssystem zunehmend vor Herausforderungen und sorgen für Passungsprobleme am Übergang (Seeber und Seifried 2019). Die KMK (2017a, 2017b) hat diese Problematik erkannt und rückt verstärkt auch die berufliche Orientierung in die Verantwortung aller allgemeinbildenden Schulformen, u. a. besonders auch des Gymnasiums. Doch wie kann ein fachlicher Beitrag zur Berufsorientierung aus dem allgemeinbildenden Mathematikunterricht heraus aussehen? Die vorgeschlagenen berufsfeldbezogenen mathematischen Kompetenzen sind ein sowohl schulisch als auch beruflich anschlussfähiges Konstrukt. Zugehörige Aufgaben mit berufsfeldbezogenen Anforderungen könnten also in den Mathematikunterricht integriert werden, da sie vereinbar mit den allgemeinbildenden Zielsetzungen der Mathematical Literacy sind. Auf Basis unserer Studie lässt sich argumentieren, dass mit der Integration solcher sich auf unterschiedliche berufliche Kontexte beziehender Aufgaben gezielt die Reichweite mathematischer Modellierungskompetenz vergrößert werden könnte. Dabei bietet es sich an, die kaufmännischen Berufe wegen ihres großen Anteils an Ausbildungsplätzen und ihres starken inhaltlichen Bezugs zur Mathematik als einen praktisch relevanten Bezugspunkt zu nutzen. Kaufmännische Kontexte können sogar dem Verständnis mathematischer Konzepte dienen, etwa wenn die lineare Abschreibung als Prototyp für einen linearen Zusammenhang verstanden wird (Lindmeier und Siebert 2015). Wie bereits zuvor erwähnt zeigt eine Schulbuchanalyse von 18 allgemeinbildenden Sekundarschulbüchern für das Fach Mathematik jedoch, dass derzeit nur ein verschwindend geringer Anteil an Lerngelegenheiten kaufmännische Perspektiven anbietet (von Hering et al. 2020). Das Potenzial zum Erwerb berufsfeldbezogener mathematischer Kompetenzen im Rahmen des allgemeinbildenden Mathematikunterrichts scheint damit noch nicht in der Breite erkannt zu sein. Die hier präsentierten Ergebnisse leisten damit auch einen Beitrag zum notwendigen Diskurs um den Stellenwert berufsbezogener Kontexte im allgemeinbildenden Mathematikunterricht.

Eine Limitation der empirischen Studie ist, dass nur drei Aufgabenpaare genutzt wurden. Auf diese Weise konnten zwar in den Stimulated-Recall-Interviews in einer angemessenen Zeitdauer alle Aufgabenpaare behandelt werden, weitere Aufgabenpaare hätten aber ggf. ein aussagekräftigeres Sampling der Personen für das Interview ermöglicht. Zudem wurde die Studie mit Industriekaufleuten in der Ausbildung von einem Standort durchgeführt. Obwohl zwei verschiedene Ausbildungsjahrgänge beteiligt waren, sind Kohorten- oder Standorteffekte nicht vollständig auszuschließen. Der zeitliche Abstand zum allgemeinbildenden Schulunterricht betrug bei allen Auszubildenden mindestens 1,5 Jahre, sodass sich die zeitliche Nähe zum beruflichen Unterricht auf die Aufgabenbearbeitungen ausgewirkt haben könnte. Gleichzeitig werden die mathematisch-inhaltlichen Anforderungen der Aufgaben mehrheitlich in Klassenstufe 7 der allgemeinbildenden Schulen behandelt und bleiben im Verlauf der Sekundarstufe relevant, so dass diese mindestens drei Jahre 
im allgemeinbildenden Mathematikunterricht genutzt wurden. Eine Stärke der Untersuchung liegt darin, dass nicht alleine die Lösungsraten der Aufgaben (also die Testperformanz) genutzt wurde, um auf die zugehörigen Kompetenzen zu schließen. Vielmehr wurden die Forschungsfragen auf Basis der Interviewstudie zu den Bearbeitungsprozessen beantwortet.

Für die Zukunft wäre anzustreben, durch eine weitere Studie mit Schülerinnen und Schülern der allgemeinbildenden Schule die Ergebnisse unter unterschiedlichen Bedingungen zu replizieren. Dazu würden idealerweise Schülerinnen und Schüler kurz vor Schulabschluss gewählt, die eine Ausbildung als Industriekaufmann/-frau anstreben. Auch Untersuchungen, die über den kaufmännischen Bereich hinausgehen und entsprechend nicht nur einen rechnerischen Schwerpunkt haben, wären relevant, um die Generalisierbarkeit der Erkenntnisse zu bestätigen. Das Konzept der berufsfeldbezogenen mathematischen Kompetenzen ließe sich hier für andere berufliche Domänen, z.B. aus dem technischen Bereich, analog anwenden. Bei einer Prüfung der Abgrenzbarkeit von allgemeinen mathematischen Kompetenzen sind dann auch geometrische statt nur algebraische Inhalte zu thematisieren.

Offen bleibt an dieser Stelle die Frage, ob die berufsfeldbezogenen mathematischen Kompetenzen tatsächlich - wie theoretisch angenommen - eine vermittelnde Rolle am Übergang von der allgemeinbildenden Schule in den Beruf spielen können. Das Projekt ManKobE (Mathematisch-naturwissenschaftliche Kompetenzen in der beruflichen Erstausbildung) untersucht in einer groß angelegten Längsschnittstudie für verschiedene Ausbildungsberufe (u.a. Industriekaufleute) die Bedingungen sowie die Entwicklung der mathematischen und beruflichen Kompetenzen im Ausbildungsverlauf und kann die theoretisch angenommene Rolle empirisch prüfen.

Funding Open Access funding enabled and organized by Projekt DEAL.

Open Access Dieser Artikel wird unter der Creative Commons Namensnennung 4.0 International Lizenz veröffentlicht, welche die Nutzung, Vervielfältigung, Bearbeitung, Verbreitung und Wiedergabe in jeglichem Medium und Format erlaubt, sofern Sie den/die ursprünglichen Autor(en) und die Quelle ordnungsgemäß nennen, einen Link zur Creative Commons Lizenz beifügen und angeben, ob Änderungen vorgenommen wurden.

Die in diesem Artikel enthaltenen Bilder und sonstiges Drittmaterial unterliegen ebenfalls der genannten Creative Commons Lizenz, sofern sich aus der Abbildungslegende nichts anderes ergibt. Sofern das betreffende Material nicht unter der genannten Creative Commons Lizenz steht und die betreffende Handlung nicht nach gesetzlichen Vorschriften erlaubt ist, ist für die oben aufgeführten Weiterverwendungen des Materials die Einwilligung des jeweiligen Rechteinhabers einzuholen.

Weitere Details zur Lizenz entnehmen Sie bitte der Lizenzinformation auf http://creativecommons.org/ licenses/by/4.0/deed.de.

\section{Literatur}

Anderson, J.R. (1996). ACT. A simple theory of complex cognition. American Psychologist, 51(4), $355-365$.

BBiG (2005). Berufsbildungsgesetz. (BBiG) in der Fassung der Bekanntmachung vom 23. März 2005 (BGBI I, S. 931).

Blomhøy, M., \& Jensen, T.H. (2003). Developing mathematical modelling competence: conceptual clarification and educational planning. Teaching mathematics and its applications, 22(3), 123-139. 
Blum, W., \& Leiß, D. (2005). Modellieren im Unterricht mit der „Tanken“-Aufgabe. Mathematik lehren, $128,18-21$.

Brown, J.S., Collins, A., \& Duguid, P. (1989). Situated cognition and the culture of learning. Educational researcher, 18(1), 32-42.

Bundesinstitut für Berufsbildung (2019). Datenreport zum Berufsbildungsbericht 2019. Bonn: BMBF.

Bundesministerium für Bildung und Forschung (2002). Verordnung über die Berufsausbildung zum Industriekaufmann/zur Industriekauffrau. Bundesgesetzblatt, 51(1), 2764-2774.

Cosmides, L., \& Tooby, J. (1992). Cognitive adaptions for social exchange. In J. Barkow, L. Cosmides \& J. Tooby (Hrsg.), The adapted mind: evolutionary psychology and the generation of culture (S. 163-228). New York: Oxford University Press.

Erpenbeck, J. (2009). Kompetente Kompetenzerfassung in Beruf und Betrieb. In D. Münk \& E. Severing (Hrsg.), Theorie und Praxis der Kompetenzfeststellung im Betrieb - Status quo und Entwicklungsbedarf (S. 17-44). Bielefeld: Bertelsmann.

Fleiss, J.L., Levin, B., \& Paik, M. C. (2003). Statistical methods for rates and proportions (3. Aufl.). New Jersey: John Wiley \& Sons.

Frank, C., Härtig, H., \& Neumann, K. (2017). Schulisch erworbene Kompetenzen als Voraussetzung für berufliches Wissen gewerblich-technischer Auszubildender. Unterrichtswissenschaft, 36, 22-35.

Heinze, A., \& Grüßing, M. (Hrsg.). (2009). Mathematiklernen vom Kindergarten bis zum Studium. Kontinuität und Kohärenz als Herausforderung beim Mathematiklernen. Münster: Waxmann.

Helfferich, C. (2011). Die Qualität qualitativer Daten: Manual für die Durchführung qualitativer Interviews. Wiesbaden: VS.

von Hering, R., Zingelmann, H., Lindmeier, A., \& Heinze, A. (2020). Lerngelegenheiten mit kaufmännischem Kontext im Mathematikunterricht der allgemeinbildenden Schule: Eine Lehrbuch- und Aufgabenanalyse. Zeitschrift für Erziehungswissenschaft, 23(1), 193-213.

Jongebloed (2011). Der Mensch als Zweck der Bildung - Übergänge zwischen allgemeinbildender und beruflicher Schule - Bestandsaufnahme sowie Probleme und Chancen. Profil - Das Magazin für Gymnasium und Gesellschaft, 18(6), 12-29.

Klieme, E., \& Leutner, D. (2006). Kompetenzmodelle zur Erfassung individueller Lernergebnisse und zur Bilanzierung von Bildungsprozessen. Zeitschrift für Pädagogik, 52(6), 876-903.

Klieme, E., Neubrand, M., \& Lüdtke, O. (2001). Mathematische Grundbildung: Testkonzeption und Ergebnisse. In PISA-Konsortium (Hrsg.), PISA 2000. Basiskompetenzen von Schülerinnen und Schülern im internationalen Vergleich (S. 139-190). Opladen: Leske + Budrich.

KMK (2003). Bildungsstandards im Fach Mathematik für den Mittleren Schulabschluss. Bonn: KMK.

KMK (2011). Handreichungen für die Erarbeitung von Rahmenlehrplänen der Kultusministerkonferenz (KMK) für den berufsbezogenen Unterricht in der Berufsschule und ihre Abstimmung mit Ausbildungsordnungen des Bundes für anerkannte Ausbildungsberufe. Bonn: KMK.

KMK (2012). Bildungsstandards im Fach Mathematik für die Allgemeine Hochschulreife. Bonn: KMK.

KMK (2017a). Empfehlung zur Beruflichen Orientierung an Schulen. Bonn: KMK.

KMK (2017b). Dokumentation zur Beruflichen Orientierung an allgemeinbildenden Schulen. Bonn: KMK.

Konferenz der Kultusminister der Länder in der Bundesrepublik Deutschland (2002). Rahmenlehrplan für den Ausbildungsberuf Industriekaufmann/Industriekauffrau. Bonn: KMK.

Lehmann, R., \& Seeber, S. (Hrsg.). (2007). ULME III. Untersuchung von Leistungen, Motivation und Einstellungen der Schülerinnen und Schüler in den Abschlussklassen der Berufsschulen. Hamburg: Behörde für Bildung und Sport.

Lindmeier, A., \& Siebert, U. (2015). Eigenkapitalquote \& Co. Mathematische Grundbildung für einen gelingenden Übergang in die kaufmännische Berufsausbildung. mathematik lehren, 192, 25-29.

Mayring, P. (2015). Qualitative Inhaltsanalyse: Grundlagen und Techniken. Weinheim \& Basel: Beltz.

Münk, D. (2013). Übergang Schule - Berufsausbildung: Strukturen im Bildungssystem - Übergangsstrukturen mit System? In G. Bellenberg \& M. Forell (Hrsg.), Bildungsübergänge gestalten. Ein Dialog zwischen Wissenschaft und Praxis (S. 251-256). Münster: Waxmann.

Neubrand, M. (2003). Mathematical literacy“/,Mathematische Grundbildung. Zeitschrift für Erziehungswissenschaft, 6(3), 338-356.

Neumann, K., Vollstedt, M., Lindmeier, A., Bernholt, S., Eckhardt, M., \& Harms, U. (2013). Strukturmodelle allgemeiner Kompetenz in Mathematik und den Naturwissenschaften und Implikationen für die Kompetenzentwicklung im Rahmen der beruflichen Ausbildung in ausgewählten kaufmännischen und gewerblich-technischen Berufen. In R. Nickolaus, J. Retelsdorf, E. Winther \& O. Köller (Hrsg.), Zeitschrift für Berufs- und Wirtschaftspädagogik Beihefte: Vol. 26. Mathematisch-naturwissenschaftliche Kompetenzen in der beruflichen Erstausbildung. Stand der Forschung und Desiderata (S. 113-137). Stuttgart: Steiner. 
Nickolaus, R. (2011). Kompetenzmessung und Prüfungen in der beruflichen Bildung. Zeitschrift für Berufs- und Wirtschaftspädagogik, 107(2), 161-173.

Nickolaus, R., \& Seeber, S. (2013). Berufliche Kompetenzen: Modellierungen und diagnostische Verfahren. In A. Frey, U. Lissmann \& B. Schwarz (Hrsg.), Handbuch berufspädagogischer Diagnostik. Weinheim: Beltz.

OECD (2000). From initial education to working life. Paris: OECD.

Perkins, D. N., \& Salomon, G. (1992). Transfer of learning. International Encyclopedia of Education, 2, 6452-6457.

PISA-Konsortium (2000). Schülerleistungen im internationalen Vergleich. Eine neue Rahmenkonzeption für die Erfassung von Wissen und Fähigkeiten. Berlin: Max-Planck-Institut für Bildungsforschung.

Przyborski, A., \& Wohlrab-Sahr, M. (2014). Qualitative Sozialforschung. Ein Arbeitsbuch. Bd. 4. München: Oldenbourg.

Ratschinski, G., Sommer, J., Eckardt, C., \& Struck, P. (2018). Berufswahlkompetenz und ihre Förderung. Evaluation des Bundesprogramms BOP. Bonn: Bundesinstitut für Berufsbildung.

Seeber, S., \& Seifried, J. (2019). Herausforderungen und Entwicklungsperspektiven der beruflichen Bildung unter veränderten Rahmenbedingungen. Zeitschrift für Erziehungswissenschaft, 22(3), 485-508.

Siebert, U., \& Heinze, A. (2016). Modellierung mathematischer Kompetenzen von Industriekaufleuten am Übergang in die berufliche Erstausbildung. In Institut für Mathematik und Informatik Heidelberg (Hrsg.), Beiträge zum Mathematikunterricht 2016 (S. 1293-1296). Münster: WTM.

Spiro, R. J., Coulson, R. L., Feltovich, P. J., \& Anderson, D. K. (1988). Cognitive Flexibility theory: Advanced knowledge acquisition in ill-structured domains. Technical Report No. $441 .$.

Weinert, F.E. (2001). Vergleichende Leistungsmessung in Schulen - eine umstrittene Selbstverständlichkeit. In F.E. Weinert \& A. Helmke (Hrsg.), Leistungsmessungen in Schulen (S. 17-31). Weinheim: Beltz.

Winter, H. (1995). Mathematikunterricht und Allgemeinbildung. Mitteilungen der Gesellschaft für Didaktik der Mathematik, 61, 37-46.

Winther, E. (2010). Kompetenzmessung in der beruflichen Bildung. Bielefeld: wbv.

Winther, E., \& Achtenhagen, F. (2008). Kompetenzstrukturmodell für die kaufmännische Bildung. Zeitschrift für Berufs- und Wirtschaftspädagogik, 104(4), 511-538.

Winther, E., \& Klotz, V.K. (2016). Berufliche Kompetenzniveaumodellierung - Eine Blaupause für die kaufmännische Domäne. Unterrichtswissenschaft, 44(2), 131-146.

Winther, E., Sangmeister, J., \& Schade, A. K. (2013). Zusammenhänge zwischen allgemeinen und beruflichen Kompetenzen in der kaufmännischen Erstausbildung. In R. Nickolaus, J. Retelsdorf, E. Winther \& O. Köller (Hrsg.), Mathematisch-naturwissenschaftliche Kompetenzen in der beruflichen Erstausbildung. Stand der Forschung und Desiderata. Zeitschrift für Berufs- und Wirtschaftspädagogik Beihefte, (Bd. 26, S. 139-157). Stuttgart: Steiner. 